\title{
THE SIMULATION OF HARD X-RAY IMAGES OBTAINED DURING LOWER HYBRID CURRENT DRIVE ON PBX-M.
}

\author{
S. von Goeler, H. Fishman, D. Ignat, S. Jones ${ }^{\dagger}$, P. Roney, J. Stevens, \\ S. Bernabei, W.Davis, R. Kaita, F. Paoletti†, \\ G. Petravich ${ }^{\dagger}$, and F. Rimini ${ }^{\dagger \dagger}$. \\ Princeton Plasma Physics Laboratory, Princeton University, \\ BOX 451, Princeton NJ. 08543
}

\begin{abstract}
During lower hybrid current drive on PBX-M suprathermal electrons in the 30 to $150 \mathrm{keV}$ range are generated. These electrons emit hard X-ray bremsstrahlung in collisions with plasma ions; the radiation creates images in a hard X-ray pinhole camera. In order to interpret the hard X-ray images, a computer simulation code has been written, the PBXRAY code. It represents an extension of the STEVENS 1 code that calculates the free-free and free-bound radiation for non-Maxwellian relativistic electron tail distributions. The PBXRAY code provides the chord integration in the bean-shaped plasma geometry on PBX-M and integrates over photon energy. The simulations show that the location of the suprathermal electrons can be determined with an accuracy of approximately two centimeters in the plasma. In particular, we analyze discharges whose characteristic "hollow" images indicate off-axis LH current drive. A comparison of images taken with different absorber foils reveals that the suprathermal electrons have less than $150 \mathrm{keV}$ parallel energy for the hollow discharges.
\end{abstract}

\section{Introduction.}

The Princeton Beta Experiment Modification PBX-M explores new ways of improving the plasma confinement and the toroidal beta (the ratio of kinetic to magnetic pressure). The goal is to find a more attractive fusion reactor concept than the standard tokamak reactor. The "beanshaped" plasma cross section of PBX-M is intended to stabilize ballooning 
modes. ${ }^{2}$ Its close-fitting stabilizer plates are designed to stabilize kink and tearing modes. Recently a $4.6 \mathrm{GHz}$ lower hybrid current drive system (LHCD) ${ }^{3}$ was installed on PBX-M for the purpose of modifying the radial current distribution in the plasma. It is projected that shaping of the current profile - in particular a lowering of the current density in the plasma center and an increase near the plasma edge - may lead to easier access to the region of "second stability" against ballooning modes.

The lower hybrid waves interact with the plasma electrons and generate a suprathermal tail, ${ }^{4}$ presumably through Landau damping. In order to investigate the physical mechanism of the interaction of the lower hybrid waves with the plasma and the suprathermal electrons, we have installed on PBX-M a hard X-ray camera5,6 that produces images of the hard X-ray bremsstrahlung generated by the suprathermal electrons. The mechanical layout of the Hard X-ray Camera is shown in Fig. 1. The hardware of the camera - the X-ray image intensifier that serves as a detector, the interfacing of the camera with the tokamak, the data acquisition system - have been described in detail in Ref. 5 and 6. A typical image obtained with the Hard X-ray Camera is shown in Fig. 2. It stems from a discharge where the suprathermal electrons are not created in the center of the plasma, but predominantly at an outer magnetic surface at about $1 / 3$ the minor radius of the plasma. We refer to such discharges as "hollow" discharges. The interpretation of the hard X-ray pictures is not straight forward, and, in order to evaluate the images in a quantitative fashion, we have written the PBXRAY simulation code. The computer program models the hard X-ray emission from the plasma: the user specifies as input a trial velocity distribution function and a trial radial profile of the suprathermal electrons, and the code generates an Xray image as output which is then compared with a measured image like Fig. 2. This procedure is iterated until a best fit to the experimental data is obtained. This paper describes the PBXRAY code and discusses what information on the physical properties of the suprathermal electrons can be obtained from the hard X-ray images.

The lower hybrid waves accelerate the electrons in a direction counter to the electric current flow. Since the electrons have relativistic energies, their bremsstrahlung emission is directed predominantly in the forward direction ${ }^{7}$ ( Fig. 3 ). The camera has been oriented in such a way that it views the plasma tangentially along the magnetic field counter to the direction of the Ohmic plasma current (Fig. 1). Thus, each pixel element on the camera input scintillator screen intercepts X-rays that are emitted along a line of sight (chord) that is determined by the location of the pixel element and the pinhole aperture. Since the plasma is optically 


\section{DISCLAIMER}

This report was prepared as an account of work sponsored by an agency of the United States Government. Neither the United States Government nor any agency thereof, nor any of their employees, make any warranty, express or implied, or assumes any legal liability or responsibility for the accuracy, completeness, or usefulness of any information, apparatus, product, or process disciosed, or represents that its use would not infringe privately owned rights. Reference herein to any specific commercial product, process, or service by trade name, trademark, manufacturer, or otherwise does not necessarily constitute or imply its endorsement, recommendation, or favoring by the United States Government or any agency thereof. The views and opinions of authors expressed herein do not necessarily state or reflect those of the United States Government or any agency thereof. 


\section{DISCLAIMER}

Portions of this document may be illegible in electronic image products. Images are produced from the best available original document. 
thin to the emitted $X$ rays, the $X$-ray intensity seen by the pixel element is the result of a line integration. For each length element on the chord, the $\mathrm{X}$-ray emission has to be computed for a model distribution of relativistic tail electrons as a function of the angle between magnetic field and sight line.

The paper is organized in the following way: In Sec. 2 we present a discussion of the atomic processes which lead to hard X-ray emission. Since the PBXRAY code uses the results from the Stevens ${ }^{1}$ code, which has been extensively documented, this discussion will be short. In Sec. 3 we report the details of the line integration and the subsequent integration over photon energy. This includes a discussion of the X-ray absorption efficiency of the scintillator screen, and the X-ray absorption by the vacuum window on the PBX-M tokamak and the entrance window of the $\mathrm{X}$-ray image intensifier. In Sec. 4 we use results from the PBXRAY code to illustrate the sensitivity of hard X-ray imaging as a diagnostic for suprathermal electrons. For instance we try to answer fundamental questions like "How well can we determine the radial location or the energy of suprathermal electrons". In Sec. 5 we show a comparison between simulation and measured hard X-ray images for a circular discharge with a peaked hard X-ray profile. In Sec. 6 we discuss a high density discharge with a hollow hard X-ray profile. Finally in Sec. 7, we present measurements of the energy of the suprathermal electrons using the absorber foil method.

\section{Atomic processes that lead to hard X-ray emission and velocity-averaged rate coefficients.}

The radiation seen by the Hard X-ray Camera usually comes from the plasma volume, i.e., it consists of electron-ion bremsstrahlung, electronelectron bremsstrahlung, and recombination radiation. As discussed in Ref. 6 , there are occasionally cases where wall radiation exists, i.e., radiation produced by suprathermal electrons that leave the plasma volume and hit limiters or other objects close to the plasma edge. However, wall radiation usually emanates only from well determined regions, namely the location of the limiters, and it usually occurs only in discharges with strong $n=1$ MHD instabilities. The following analysis considers only volume radiation.

For the calculation of the atomic processes we use the Stevens code, 1 which was written in 1983 for the interpretation of lower hybrid 
experiments on the PLT tokamak. The code calculates the hard X-ray emission as a function of photon energy and angle $\theta$ between the electron velocity and the direction of photon emission for electrons of energy $E$. It then sums the contributions from all the electrons of a given distribution function. The model velocity distribution function of the suprathermal electrons is an input to the code. Usually we choose a Gaussian determined by four parameters: the parallel forward temperature $T_{\| f}$, the perpendicular temperature $T_{\perp}$, the parallel backward temperature $T_{\| b}$, and a high energy cutoff $\mathrm{E}^{*}$, or the corresponding momentum $\mathrm{p}^{*}$,

$$
\begin{array}{rlrl}
f(\vec{p}) & =\text { const } \times \exp \left(-\frac{p_{\perp}^{2}}{2 m k T_{\perp}}-\frac{p_{\|}^{2}}{2 m k T_{\| f}}\right) & & \text { for } p_{\|} \geq 0 \\
& =\text { const } \times \exp \left(-\frac{p_{\perp}^{2}}{2 m k T_{\perp}}-\frac{p_{\|}^{2}}{2 m k T_{\| b}}\right) & & \text { for } p_{\|}<0 \\
& =0 & \text { for } p_{\|}>p^{*} .
\end{array}
$$

Although this distribution is arbitrary, it can be made to resemble theoretical predictions 8,9 that have been made for lower hybrid waves on the basis of computations with the Fokker-Planck equation. In essence, the distribution features (1) a slope $\left(T_{\| f}\right)$, which depends on the quasilinear diffusion of the tail electrons in the electric fields of the RF waves, (2) a high-energy cutoff $\left(E^{\star}\right.$ or $\left.p^{\star}\right)$ related to the low $n_{\|}$cutoff of the launched RF spectrum, and (3) a perpendicular and a backward spread $\left(T_{\perp}, T_{\| b}\right)$ that depend on the perpendicular Coulomb scattering. An example for a typical velocity distribution function, which will be used later to simulate a hollow discharge, PBX-M shot \#298069, is given in Fig. 4. Direct modeling of hard X-ray images from Fokker-Planck calculations using the Lower Hybrid Wave Simulation CODE (LSC) and the GIRRUZI CODE has also been performed.10,16

The atomic processes that are included in the computation are electron-ion bremsstrahlung, electron-electron bremsstrahlung, and recombination radiation. For the electron-ion bremsstrahlung, which gives the dominant contribution to the hard X-ray emission (except for low values of $T_{\| f}$ ), the Stevens code uses Born approximation cross sections ${ }^{11}$ with a Coulomb correction factor derived by Elwert.12 These are believed to be accurate to within 10\%. For electron-electron bremsstrahlung cross sections the code uses a Born approximation formula by Haug, ${ }^{13}$ again multiplied by an Elwert factor. The recombination radiation cross sections of the code are derived from relativistic Born-approximation K-shell photo ionization cross sections using a detailed balancing argument. The 
contribution from recombination radiation, which depends on impurity content $\left(\mathrm{Z}_{\mathrm{eff}}\right)$ and the details of the charge state distribution of the relevant impurity ions, represents a relatively large uncertainty for low values of $\mathrm{T}_{\text {II }} \mathrm{f}$, because the exact impurity composition is not known for most of the plasma shots presented here. In all computations we have set $\mathrm{Z}_{\mathrm{eff}}=5$ and have assumed that the dominant impurity is carbon. The choice of carbon makes electron-ion bremsstrahlung the dominant X-ray emission process even for $\mathrm{T}_{\| \mathrm{f}}$ as low as $5 \mathrm{keV}^{14}$.

Since the calculation of the hard X-ray emission from an arbitrary velocity distribution function uses a large amount of computer time, we have separated the atomic calculations from the rest of the computer program. Specifically, we have slightly modified the Stevens code so that it writes a file that contains a $12 \times 14$ matrix for the X-ray intensity: the 12 rows correspond to the photon energy and the 14 columns to the angle $\theta$ between the sight line and the magnetic field. We have established a small library that contains these files for a variety of values of $T_{\| f}, T_{\perp}, T_{\| b}$, and $E^{*}$. The library is accessed by the PBXRAY code when it is executed.

\section{Line integration through the plasma.}

The shape of the distribution function is not the only input that the PBXRAY code needs. We also have to specify the plasma geometry and the radial profile for the suprathermal electrons.

Let us begin with the plasma geometry. The shape of the plasma is determined with the FQ code, which computes the plasma equilibrium for a PBX-M shot from measured signals, i.e., from magnetic pick-up coils, magnetic flux loops, Rogowski coils, and from Thomson scattering data (pressure profiles). The FQ code was modified to generate a special file that provides information on (a) the magnetic flux function $\Psi$ [normalized to zero in the plasma center and to one at the limiter (or at the separatrix, if a separatrix exists inside the limiter)], (b) the plasma density [the density is set to zero outside the separatrix or the last closed flux surface] and (c) the three components of a unit vector in the direction of the magnetic field. Each of these five quantities is specified as a $65 \times 65$ matrix in the $(R, z)$-plane of the tokamak, where $R$ is the major radius and $z$ the height above the mid plane of the tokamak. A contour plot of a typical 
flux function ( $\Psi$ ) for discharge \#298069 - for which we provide a detailed analysis later in Section 6 - is shown in Fig. 5.

Another input variable is the radial density profile of suprathermal electrons. We assume for simplicity that the radial profile is a Gaussian function of the magnetic flux $\psi$, i. e.,

$$
\begin{array}{ll}
n_{s e}=n_{s e 0} \frac{R_{0}}{R} \exp \left[-\left(\frac{\psi-\psi_{s e}}{\Delta \psi_{s e o}}\right)^{2}\right] & \text { for } \quad \psi \geq \psi_{s e} \\
n_{s e}=n_{s e 0} \frac{R_{0}}{R} \exp \left[-\left(\frac{\psi-\psi_{s e}}{\Delta \psi_{s e i}}\right)^{2}\right] & \text { for } \quad \psi<\psi_{s e}
\end{array}
$$

where $\Psi_{\text {se }}$ is the magnetic surface where the suprathermal electron density has a maximum. The quantity $\Delta \Psi_{\text {seo }}$ determines the fall-off of the suprathermal density toward the outside, and the quantity $\Delta \Psi_{\text {sei }}$ determines the fall-off towards the plasma center, i.e., from the surface $\Psi_{\text {se }}$ where the maximum is located towards the magnetic axis for hollow discharges. The factor $R_{0} / R$ ( $R=$ major radius, $R_{0}$ major radius of the magnetic axis) tries to approximate poloidal variations of the density of suprathermal electrons. For a beam of suprathermal electrons that consists only of passing particles, the particle flux $d \mathrm{~N} \mathrm{v}_{\|}$is approximately conserved along a magnetic flux tube $d \Psi=B d A$, where $d N$ is the number suprathermal electrons per unit length in the flux tube of cross-sectional area dA. In the special case $v_{\perp}=0$, the parallel velocity $v_{\|}$does not vary in poloidal direction, and the density of suprathermal electrons $n_{s e}$ becomes proportional to the magnetic field. For passing particles with $v_{\perp} \neq$ 0 , there will be deviations from the $1 / R$ dependence; since the parallel velocity $v_{\|}$is larger for bigger $R$, the density of suprathermal electrons decreases towards the outside even stronger than $1 / R$. At the other extreme, for an isotropic Maxwellian distribution, we expect that $\mathrm{n}_{\text {se }}$ becomes a surface quantity and the factor $R_{0} / R$ should be left out in Eq.(2). As a compromise, we have assumed in all calculations a $1 / R$ dependence. However, we want to point out that large deviations from the $1 / R$ dependence may occur in the presence of large numbers of trapped suprathermal electrons, and that there are indications that this situation sometimes actually occurs. ${ }^{18}$ We consider this the point of greatest uncertainty in the modeling of the Hard X-ray images, and we have, at the present time, no simple way of estimating the trapped particle fraction of the suprathermal electrons. An example for a suprathermal density profile, which will be later used to simulate a hollow discharge, PBX-M shot \#298069, is shown in Fig. 6. 
The computation, as we have outlined it up to now, does not allow for variations of the distribution function, for instance the parallel forward temperature $T_{\| f}$, with minor radius. In order to provide some flexibility in this respect, we have added a loop in the code that allows to repeat the calculation several times with different distribution functions $\left(f_{1}, f_{2}, f_{3}, \ldots\right)$, that are centered on different magnetic flux surfaces ( $\left.\Psi_{\text {se1 }}, \Psi_{\text {se2 }}, \Psi_{\text {se3 }}, \ldots ..\right)$ and have different fall-off distances $\left(\Delta \Psi_{\mathrm{se} 1}, \Delta \Psi_{\mathrm{se} 2}, \Delta \Psi_{\mathrm{se} 3}, \ldots ..\right)$. The X-ray intensities for all the various distribution functions are added. In this way, at least some preliminary information can be obtained on the effect of changing the distribution function of the suprathermal electrons (or quantities like $T_{\| f}$ or $T_{\perp}, T_{\| b}$, and $E^{*}$ ) as a function of minor radius. Figure 6 , which represents the radial distribution for a typical hollow discharge (PBX-M shot \#298069), illustrates this procedure: The hot electron component consists predominantly of a cool component with $\mathrm{T}_{\| \mathrm{f}}=100$ $\mathrm{keV}$. However, as we shall discuss in Sec. 7, the absorber foil measurements are best explained if we assume the presence of a small hotter population of suprathermal electrons with $\mathrm{T}_{\| \mathrm{f}}=400 \mathrm{keV}$ near the plasma edge.

Let us now discuss the line integration itself. The screen of the camera is usually simulated by a $50 \times 50$ pixel grid. (The axes are often labeled as for a $100 \times 100$ pixel image in order to facilitate the comparison with the experimental images). For each pixel a line of sight through the plasma is established in an R, z, $\phi$ coordinate system, where R is the major radius, $z$ the vertical distance from the tokamak mid plane, and $\phi$ the toroidal angle. The tokamak has an outer limiter at $\mathrm{R}=197 \mathrm{~cm}$. Integration starts and stops at this major radius as the line of sight enters and leaves the plasma region. The chord length in the plasma is divided into 100 steps. At each step the code calculates:

(1) the $\mathrm{z}$ and the $\mathrm{R}$ coordinates and checks for interference of the chord with the PBX-M pusher coil or the PBX-M stabilizer plates,

(2) a unit vector of the sight line in the $R, z, \phi$ coordinate system,

(3) the unit vector of the magnetic field by interpolating the three $65 \times 65$ matrices from the equilibrium file,

(4) the angle between sight line and magnetic field, and

(5) the velocity averaged cross section of the X-ray emission in direction of the chord by interpolating the $12 \times 14$ matrix for the bremsstrahlung emission from the library file, 
(6) the product of the velocity averaged cross section with the density of suprathermal electrons, with the plasma density from the equilibrium file, with the length of the line element, and with the impurity factor $\mathrm{Z}_{\mathrm{eff}}$.

The result is then a set of twelve $50 \times 50$ images for various photon energies $(10,20,30,40,50,60,80,100,150,200,250$, and $300 \mathrm{keV})$.

Now one still has to perform an integration over photon energy, since the output of the X-ray image intensifier tube is proportional to the X-ray photon energy absorbed in the input scintillator screen. For this purpose a detection efficiency function 6 is calculated that takes into consideration (1) the X-ray absorption in the $3 \mathrm{~mm}$ thick Al vacuum window on PBX-M, (2) the X-ray absorption in the $1 \mathrm{~mm}$ thick $\mathrm{Al}$ entrance window of the image intensifier, (3) the X-ray absorption in the $0.2 \mathrm{~mm}$ thick CONNECTIC foil that has been used for magnetic shielding, (4) the X-ray absorption in one of the optional absorber foils, and finally (5) the absorption efficiency of Xrays in the $0.3 \mathrm{~mm}$ thick CsI scintillator of the Hamamatsu X-ray image intensifier. The absorption coefficients for these calculations have been taken from the MacMaster tables ${ }^{15}$. The integration over energy is then performed by folding the X-ray spectrum with the detection efficiency function. The final result is a computer-generated image that can be compared with experimental images from the Hard X-ray Camera.

\section{The sensitivity of hard X-ray images to changes of plasma parameters.}

In this section we want to discuss how the images of the Hard X-ray Camera respond to changes in plasma parameters, according to the predictions of the PBXRAY code. On PBX-M, for example, we would like to increase the plasma current at the plasma edge. If the lower hybrid waves produce suprathermal electrons in this region, the Hard X-ray Camera must be able to differentiate between edge electrons and central electrons. The subjects that we want to discuss in this section are (1) the radial location of the suprathermal electrons, (2) the energy of the suprathermal electrons, (3) the isotropy of the suprathermal electrons, and finally (4) helical effects. The latter effects are produced by the fact that the suprathermal electrons run along magnetic field lines encircling the magnetic axis of the plasma in a helical fashion. 
Let us first discuss the capability of the camera to determine the radial location of the suprathermal electrons. In Fig. 7 we show four hard $X$-ray images that have been computed with the PBXRAY code. In the computation the flux surface $\Psi_{\text {se, }}$, where the density of suprathermal electrons $\mathrm{n}_{\mathrm{se}}$ has a maximum, is varied from 0.0 in subfigure (a) to $\Psi_{\mathrm{se}}=$ $0.25,0.50$, and 0.75 in subfigures (b), (c), and (d), respectively. Here, $\Psi=$ 0 is the magnetic axis and $\Psi=1$ the plasma edge [limiter, separatrix]. The velocity distribution function is the same in all four images, $\left(T_{\| f}=100\right.$ $\mathrm{keV}, \mathrm{T}_{\perp}=10 \mathrm{keV}, \mathrm{T}_{\| \mathrm{b}}=10 \mathrm{keV}$, and $\mathrm{E}^{\star}=500 \mathrm{keV}$ ), as were the fall-off distances, $\Delta \Psi_{\text {sei }}=\Delta \Psi_{\text {seo }}=0.2$. The equilibrium file corresponds to a bean shaped plasma (shot 298069 shown in Fig. 5). When the suprathermal electrons are located in the center $\left(\Psi_{\text {se }}=0\right)$, the X-ray intensity contours in the center of the image are distorted circles [subfigure (a)]. Because the image is the result of a chord integration, the sight line where the maximum of the intensity occurs [pixel $(59,50)$ ], is located to the left of the sight line that is tangential to the magnetic axis. That sight line [pixel $(66,50)]$ is marked by a cross $\times$. A little further out the contour lines bulge out to the left, i.e., towards the smaller major radius side, as one would expect looking tangentially through a doughnut. The steep descent near pixel $(31,50)$ is caused by interference with the pusher coil. In the region to the left from the descent, the plasma behind the pusher coil is obscured from view. When the suprathermal electrons are located further outside in a shell around the magnetic axis, as in subfigures (b), (c), and (d), the Xray image takes on a crescent or double sickle shape. This characteristic shape becomes more pronounced, when the shell is located further out and more hollow, as in subfigure $c$ and $d$. The crescent shape is easily explained as the result of chord integration. In the horizontal mid plane, we consider two sight lines that go right through the hot electron shell. One of them goes through the shell to the right of the magnetic axis, the other one to the left of the magnetic axis. The right sight line intersects the emitting region only one time, whereas the left sight line intersects the hot electron shell three times. Therefore, the intensity of the left sight line will be stronger than the intensity of the right sight line. The hollowness of the discharge is immediately evident because of the deep trough in X-ray intensity somewhat to the left of the magnetic axis at pixel $(66,50)$. The largest X-ray intensity actually occurs above or below the mid plane near the end of the sickle. These are sight lines that have the longest path length through the shell, because the projection of the sight line in a poloidal plane is tangential to the shell. We also point out that the vertical height and the horizontal width of the four images change dramatically for the four images, so that we believe that one can determine the radial location of suprathermal electrons extremely well. 
There are, however, certain caveats: First it is clear that this problem is similar to an Abel inversion. (In the tokamak mid plane it is actually exactly an Abel inversion problem). For an Abel inversion, it is known that the density inside the hollow shell is usually determined with much less accuracy, and we expect this to be true also for our case. Second, we shall discuss later that the X-ray intensity depends strongly on the energy of the suprathermal electrons. Therefore, electrons with very high energies on the outside of the plasma can dominate the radiation from slow electrons that are located in the center of the plasma. Otherwise, the camera can measure the radial location of the suprathermal electrons with very good accuracy.

Let us now discuss how the properties of distribution function affect the hard X-ray images. First we vary the energy of the suprathermal electrons, i.e., we change the parallel forward temperature $T_{\| f}$, keeping the ratios $T_{\perp} / T_{\| f}, T_{\| b} / T_{\| f}$, and $E^{\star} / T_{\| f}$ fixed. Figure 3 shows that the X-rays are emitted more in forward direction for high energy electrons, and we consequently expect that the total X-ray intensity increases with increasing $T_{\| f}[\operatorname{effect}(\mathrm{a})]$, and we also expect that those parts of the image where the sight lines are tangential to magnetic field lines will be enhanced [effect (b)]. Figure 8 shows the variation of the maximum X-ray intensity of the computed image vs. $T_{\| f}$ for four cases using different foil absorbers. There is a strong increase of the X-ray intensity with the parallel forward temperature $T_{\| f}$ [effect (a)], and an even larger increase is observed when an absorber foil is placed in the X-ray path. It should be pointed out the curves in Fig. 8 incorporate the efficiency of the X-ray image intensifier, which decreases fairly rapidly for higher energy X-rays. Figure 9 illustrates effect (b). It shows horizontal profiles for four different values of $\mathrm{T}_{\| \mathrm{f}}$. (Horizontal profile is our nomenclature for a horizontal slice through the center of an image). In order to facilitate comparison between the curves, the maxima of the images were normalized to one. A characteristic feature associated with the forward emission is the fall-off of the X-ray intensity at the left side of the curves, which becomes steeper for higher values of $T_{\text {IIf. }}$. The pixels on the left side of image have sight lines that cross the magnetic field at large angles, whereas the sight line in the middle and on the right side of the image are almost tangential to the magnetic field lines. Therefore, Figure 9 demonstrates effect (b).

The variations in the fall-off of the X-ray horizontal profile are actually caused by the anisotropy of the tail electron distribution. The slope will also change if one varies the perpendicular temperature $T_{\perp}$ and 
keeps the parallel forward temperature $\mathrm{T}_{\| \mathrm{f}}$ fixed. This behavior is demonstrated by the horizontal profiles plotted in Fig. 10. The three curves have the same $T_{\| f}$, but different $T_{\perp}=T_{\| b}$. The variation in the falloff of the horizontal profile shown in the figure is comparatively large because a large parallel forward temperature, $T_{\| f}=400 \mathrm{keV}$, was chosen. For smaller values of $T_{\| f}$, the variation would be not so large. Since the steepness of the fall-off of the horizontal profile at small major radius can be due to either anisotropy or electron energy, we cannot use it to determine the energy of the suprathermal electrons. We obtain $\mathrm{T}_{\| \mathrm{f}}$ from absorber foil measurements, which will be discussed in Sec. 6 .

Finally, we want to briefly mention helical effects. These are not important for our PBX-M measurements, because the suprathermal electrons produced by the LH-waves have comparatively low energies and relatively broad X-ray emission cones. However helical effects could become important for very high energy electrons in the $\mathrm{MeV}$ range. In order to gain some understanding, let's assume a very simple model where the suprathermal electrons would have infinite energies, so that their emission would be needle sharp in forward direction, i.e., parallel to the magnetic field. (For simplicity, we further assume that the mass of the electrons remains so small that the electrons still stick approximately to magnetic surfaces.) As the sight line travels through the plasma, it crosses magnetic surfaces, first going in and then going out. It is tangent only to the innermost surface, and, if we assume that X-ray emission occurs only in direction of the magnetic field, the sight line can receive a contribution to the X-ray signal only from the surface that contains the tangency point. (This argument is not strictly correct, because theoretically some sight lines can travel towards the magnetic axis, then away from the axis, then towards and away from the axis again. In this example, there would be three points along the sight line which can contribute to the X-ray emission.) At the tangency point, the sight line would have a local q value. [We define the local q-value as the ratio $\delta \phi / \delta \theta$, where $\delta \phi$ and $\delta \theta$ are the change of the toroidal angle or the poloidal angle as one moves a distance $\delta 1$ along the sight line]. This means an X-ray contribution to a sight line can occur only if by accident the local $q$ of the magnetic field agrees with the local q of the sight line. Figure 11 shows the local q at the tangency points of sight lines for a circular plasma. Since the toroidal magnetic field in PBX-M is directed opposite to the plasma current, we expect to see X-rays, for extremely energetic electrons, only in the upper half of the picture, where the local $\mathrm{q}$ of the magnetic field is positive. If the X-ray emission were sufficiently sharp, hard X-ray imaging could provide a direct qmeasurement. In Fig. 12 we show an image that was calculated for a 
circular plasma in PBX-M with $\mathrm{T}_{\| \mathrm{f}}=999 \mathrm{keV}, \mathrm{T}_{\perp}=50 \mathrm{keV}, \mathrm{T}_{\| \mathrm{b}}=50 \mathrm{keV}$, and $\mathrm{E}^{\star}=999 \mathrm{keV}$. The equilibrium for the calculation came from a circular experimental discharge (shot \#291428). The picture exhibits an up-down asymmetry, and, as one would expect from the simple model, it is the upper half of the image that is enhanced. However, it is clear that the peaking of the x-ray emission, even for this comparatively energetic electron distribution, is insufficient to concentrate all the emission in the region where the $\mathrm{q}$ of the sight line equals the $\mathrm{q}$ of the magnetic surfaces. Therefore, it is unlikely that the helical effects can be used to perform q measurements. Nevertheless, it is also evident from the figure that one should expect to see small up-down asymmetries in X-ray images from very energetic hot electron distributions, e.g., from run-away electrons.

\section{A circular low density discharge.}

In the following two sections we show examples of the application of the PBXRAY code. We start by discussing images from a circular low density discharge, PBX-M shot \#291430. The time dependence of the plasma current, the plasma line density, the lower hybrid power, and signal of two soft X-ray diodes are shown in Fig. 13. The plasma current is about $110 \mathrm{kA}$, and the average plasma density is circa $8.0 \times 10^{12} \mathrm{~cm}^{-3}$. $300 \mathrm{~kW}$ net $\mathrm{LH}$ power with - $90^{\circ}$ phasing is applied in the time interval from 350 to $550 \mathrm{~ms}$. For circular plasmas at these low densities a considerable reduction of the loop voltage (not shown) is observed; the one-turn voltage falls from $1.5 \mathrm{~V}$ before the lower hybrid to approximately $0.5 \mathrm{~V}$ at the end of the lower hybrid. However, although the loop voltage is reduced, it does not go to zero or become negative, and can still lead to some electron runaway. Also shown is the central trace and an outer trace of the horizontal soft X-ray diode array. Forty milliseconds after the lower hybrid is switched on, large sawtooth oscillations appear that suggest that the rf power is deposited in the central plasma region and drives some current there, which causes a lowering of the safety factor $\mathrm{q}(0)$ on axis below 1. A contour plot of a typical hard X-ray image is shown in Fig. 14a. In PBX-M, the plasma is usually centered between the armor of the pusher coil $(R=1.342 \mathrm{~m}$, pixel 31) and an outside limiter $(R=1.97 \mathrm{~m}$, pixel 102). The magnetic axis is therefore located at a major radius $R_{0}$ of $1.66 \mathrm{~m}$ (pixel 64) for well-centered low-beta plasmas, and at a somewhat larger radius for higher beta plasmas, depending on the Shafranov shift. The PBX-M plasma equilibrium code predicts the magnetic axis for shot 291430 to be 
located at $\mathrm{R}=170 \mathrm{~cm}$, i.e., at pixel 69 . One sees immediately that the shadow of the pusher coil appears at the expected position (pixel 31). On the other hand, the brightest spot of the image does not occur where the magnetic axis is, but considerably further inside near pixel 55 . This happens, of course, because of the integration along sight lines, as discussed for Fig. 7a. For circular plasmas the height of the plasma is comparable to its width, and, therefore, the vertical extent of the hard Xray image is much smaller than for bean shaped plasmas (e.g., Fig. 2). In Fig. 14b we show the results of a best-fit simulation of shot 291430 with the PBXRAY code. In the simulation we assumed that suprathermal electrons with $T_{\| f}=200 \mathrm{keV}, T_{\perp}=T_{\| b}=50 \mathrm{keV}$, and $E^{*}=500 \mathrm{keV}$ have the radial profile $\mathrm{n}_{\mathrm{se}}$ shown in Fig. 16. The value for the parallel forward temperature was chosen on the basis of absorber foil measurements, which will be discussed in Sec. 7. The radial profile is made up of two Gaussians which have the same velocity distribution functions. A better comparison between measurement and simulation is obtained by plotting the measured and simulated horizontal and vertical profiles on top of each other, as shown in Fig. 15. The second Gaussian is needed to simulate the relatively broad wing (or pedestal) of the vertical profile. The little hump in the vertical profile at pixel 78 is a hot $\operatorname{spot}^{6}$ caused by wall radiation from one of the graphite bars which protect the PBX-M stabilizer plates. The small wiggles in the experimental profiles, e.g., the minimum in the vertical profile at pixel 44 , are caused by a lead wire grid 6 that is placed in front of the X-ray imaging tube in order to check the extent to which the image is distorted by the magnetic field. The wiggles are even larger in the raw data, and have been reduced here by smoothing. The simulation fits the experimental data within 10\%, and, therefore, we believe that Fig. 16 represents the true spatial distribution of the suprathermal electrons. Most of the hot electrons are found right in the center of the discharge. In PBX-M, such centrally peaked profiles are typical for discharges at low plasma densities, i.e., $\left\langle\mathrm{n}_{\mathrm{e}}\right\rangle \cong 1.5 \times 10^{13} \mathrm{~cm}^{-3}$ and smaller. We believe that the suprathermal electrons created by the LH waves run away in the Ohmic electric field in these discharges. There are usually no hard X-rays before the onset of the rf power. The hard X-ray profile for the first few milliseconds is usually much broader than later in time, and the profile peaks up and increases in intensity continuously during the 100 or 200 milliseconds that the rf is applied (Fig. 17). After the rf is switched off, the hard X-ray intensity decreases only slowly, and in some cases it even increases. The foil measurement, which we shall discuss in Sec. 7 , shows that the energy of the suprathermal electrons increases continuously during the $\mathrm{LH}$, and the value for the forward parallel temperature indicates that the velocity of the electrons is much larger than the parallel 
phase velocity of the injected LH waves, i.e., that the acceleration is by the electric field and not by the waves. This situation is fairly typical for PBX$M$, except that Coulomb collisions provide enough friction to prevent the electron run away region at higher density, i.e., for line averaged densities $<\mathrm{n}_{\mathrm{e}}>$ above $\cong 1.7 \times 10^{13} \mathrm{~cm}^{-3}$. In that region we observe hollow profiles, as discussed in Sec. 6 .

\section{A Bean-Shaped High Density Discharge.}

In this section we want to discuss PBX-M shot \#298069, a discharge with a hollow hard X-ray radiation profile. The plasma current for this shot was $I_{p}=190 \mathrm{kA}$ and the plasma density $n_{e}=1.6 \times 10^{13} \mathrm{~cm}^{-3}$ (Fig. 18). LHCD with $-90^{\circ}$ phasing and $185 \mathrm{~kW}$ of power took place from time 250 $\mathrm{ms}$ to time $650 \mathrm{~ms}$. Simultaneously, 2.2 MW of neutral beam were injected. As soon as the RF started, the hard X-ray emission was observed. The hard X-ray vertical profile was very hollow initially; a contour plot of the X-ray image from the early stage is shown Fig. 19a, representing an average of 5 frames from time 270 to $295 \mathrm{~ms}$. The image exhibits the characteristic crescent-like (or double-sickle) shape that is typical for "hollow" discharges on PBX-M, as discussed in connection with Fig. 7c. For comparison we show a best-fit computer simulation with the PBXRAY code in Fig. 19b. As we pointed out before, overlays of computed and measured horizontal and vertical profiles are often better suited for a quantitative comparison. These are shown in Figs. $20 \mathrm{a}$ and $20 \mathrm{~b}$. The experimental data are represented by the solid curves, the computer simulations by the dotted lines. A comparison between Figs. 20 and 15 shows clearly the difference between peaked and hollow profiles: The vertical profile of hollow discharges has two distinct maxima at pixels 32 and 55, and the horizontal profile a distinct shoulder at pixel 74 . The agreement between the measured profiles and the computed profiles is again excellent. The velocity distribution of the suprathermal electrons, that went into the simulations, was again chosen on the basis of absorber foil measurements, and is characterized by a forward parallel temperature of $\mathrm{T}_{\| \mathrm{f}}=100 \mathrm{keV}$, a perpendicular temperature of $\mathrm{T}_{\perp}=\mathrm{T}_{\| \mathrm{b}}=10 \mathrm{keV}$, and a cut off energy $\mathrm{E}^{*}=$ $500 \mathrm{keV}$. As we will discuss in more detail in Sec. 7, there was also a higher energy component $\left(T_{\| f}=400 \mathrm{keV}, T_{\perp}=T_{\| b}=50 \mathrm{keV}, E^{*}=999 \mathrm{keV}\right)$, but it constituted only a small fraction of the total number of suprathermal electrons. The radial profile $\mathrm{n}_{\mathrm{se}} \mathrm{used}$ for the simulation was already shown in Fig. 6 . The hollowness of $n_{s e}$ is much deeper than the hollowness 
of the vertical profiles, which is the case because the vertical profiles are chord integrated. The energetic electron component is very small, and it is confined to the plasma edge region. The hard X-ray profiles for shot 298069 stayed hollow throughout the whole rf phase, and the intensity of the hard X-rays saturated after about $30 \mathrm{~ms}$ (see Fig. 21). The initial hollowness is larger than later in time, a behavior that was observed in many shots. In hollow discharges, the X-ray intensity decreases very rapidly after the rf is turned off. (This is not shown in Fig. 21, where the hard X-ray measurement stops at $555 \mathrm{~ms}$ before the LH power is turned off.) The value of $100 \mathrm{keV}$ for $\mathrm{T}_{\| \mathrm{f}}$ is comparable to the approximately 75 $\mathrm{keV}$ energy which electrons have that travel with a speed close to the parallel phase velocity of $\mathrm{LH}$ waves at $-90^{\circ}$ phasing. We consequently believe that the hot electrons are not created, in this case, in a run-away type of process, but are accelerated by the LH waves.

\section{Determination of the Energy of the Suprathermal Electrons by the Absorber Foil Method.}

The absorber foil method is well known from soft X-ray measurements. Images from two plasma shots are taken, the first without foil, the second with an absorber foil placed into the X-ray path. The intensity of the two images is then compared, and a photon temperature, or more exactly an "effective temperature of the photon spectrum", $\mathrm{T}_{\text {photon, }}$ is determined

$$
T_{\text {photon }}=\frac{E_{\text {foil } 2}-E_{\text {foil }}}{\ln \left(I_{\text {foil } 1}\right)-\ln \left(I_{\text {foil } 2}\right)}
$$

where $E_{\text {foil1 }}$ and $E_{\text {foil2 }}$ are the low energy cutoffs for foil \#1 and foil \#2, respectively, and $I_{\text {foil } 1}$ and $I_{\text {foil } 2}$ are the $X$-ray intensities of the first and the second image. If the spectrum of the emitted bremsstrahlung falls off with energy like an exponential function, and if the cutoffs are very sharp, then $\mathrm{T}_{\text {photon }}$ is the negative reciprocal slope of the spectrum in a semilog plot. Tphoton is determined for each pixel, and the result is an image of the photon temperature. The absorber foils consist of copper $(0.52 \mathrm{~mm})$, molybdenum $(0.95 \mathrm{~mm})$, and silver $(2.03 \mathrm{~mm})$. Without any absorber foil, the $2 \mathrm{~mm}$ aluminum vacuum window, the $1 \mathrm{~mm}$ aluminum entrance window of the X-ray image intensifier tube, and the $0.2 \mathrm{~mm}$ connectic magnetic shielding foil in front of the imaging tube contribute to a low 
energy cutoff of $45 \mathrm{keV}$. The cutoff energies with the additional $\mathrm{Cu}, \mathrm{Mo}$, and $\mathrm{Ag}$ foils are $67 \mathrm{keV}, 115 \mathrm{keV}$, and $176 \mathrm{keV}$, respectively.

An example of a photon temperature measurement is shown in Fig. 22a. Plasma shot \#298070 is nearly identical to shot \#298069, except that a copper $(\mathrm{Cu})$ foil was placed in front of the pinhole of the Hard X-ray Camera. Figure $22 \mathrm{a}$ is a contour plot of the photon temperature determined from those two shots, averaged over 5 frames in the time interval 270 to $295 \mathrm{~ms}$. The photon temperature is approximately $25 \mathrm{keV}$ in the central region of the discharge, and it rises to about $40 \mathrm{keV}$ towards the outside, large-major-radius-side of the image. Near the plasma edge, the X-ray intensity is very small, and the measurement was truncated when the signal became smaller than 3 bits. This truncation has to be performed, in order to avoid singularities in the denominator of Eq. (3). There is a question, of course, where to truncate, and how realistic the observed increase of the photon temperature near the plasma edge is in view of the uncertainties. We have examined the raw data carefully and found that a small pedestal develops in shot \# 298070 in the time interval 10 to $20 \mathrm{~ms}$ after the start of the rf. Therefore, we think that the higher edge photon temperature is real. Also a shot with a molybdenum foil exists (plasma shot \#298071). The photon temperature determined from the no-foil (nf) shot and the molybdenum shot looks very much like Fig. 22a. The hard X-ray intensity is much weaker for the Mo shot, however, and the measurement has to be truncated further inside. In Fig. 23a, we show horizontal profiles of the photon temperature for the nf- $\mathrm{Cu}$ case and the nf-Mo case. The nf-Mo case gives a slightly higher photon temperature.

In order to interpret the photon temperature measurements in terms of a hot electron distribution function, we have simulated the photon temperature with the PBXRAY code, i. e., we have simulated first an image for the no-foil case, then an image for the copper case. Then we subjected the simulated images to the same algorithm that we used for the experimental images in order to determine the photon temperature. The simulated photon temperature is shown in Fig. 22b and in Fig. 23b. The simulations agree quite well in magnitude with the measurements, and they reproduce nicely the rise of the photon temperature on the right (large major radius) side of the image. However, we have no explanation for the small increase of the photon temperature on the left side of the image. The distribution functions and the radial profiles of suprathermal electrons $\mathrm{n}_{\mathrm{se}}$, which we used for the simulations, have been shown already in Fig. 4 and in Fig. 6, respectively. It was not possible to simulate the photon temperature with only one distribution function. We had to use a 
second energetic hot electron component in order to simulate the increase of the photon temperature near the plasma edge. Figure 24 shows horizontal photon temperature profiles calculated with only one distribution function. In this case the horizontal profiles are very flat, i. e., the photon temperature does not change appreciably across the image, - in spite of the fact that for instance the angle with respect to the magnetic field changes appreciably across the image. This indicates that the photon temperature measurement really is sensitive to radial variations of the hot electron distribution. On the other hand, Fig. 24 shows that the photon temperature changes only by a factor two, whereas the parallel forward electron temperature changes by a factor 10 . This indicates that the photon temperature measurement is very sensitive to perturbations. For instance, if the two shots from which the photon temperature is derived are not exactly identical, the photon temperature is wrong. We found in particular that the number of suprathermal electrons depends very sensitively on the plasma density, and in cases where the density for the two plasma shots is not exactly the same, the photon temperature measurement becomes meaningless. This is a also a problem for discharges where the photon temperature is high, because there the difference between Ifoil1 and Ifoil2 becomes small. We have in the meantime installed a new foil drive that allows foil measurements on the same plasma shot and gives better results.

The analysis is done not only for one frame, but for a whole shot. In Fig. 25, we show the photon temperature in the central plasma region as a function of time for the $\mathrm{nf}-\mathrm{Cu}$ case and the nf-Mo case. At time $\mathrm{t}=400 \mathrm{~ms}$ the photon temperature for the nf-Cu case seems to drop, whereas the nfMo temperature stays unchanged. At the time of the drop the plasma density for shot \#298070 (the $\mathrm{Cu}$ shot) starts to deviate by $5 \%$ from the density for the other two shots. Therefore, we think that the drop is not real, but reflects the sensitivity of the absorber foil method to density variations. A similar analysis of the photon temperature was also done for the low density shot \#291430 and is shown in Fig. 26. The photon temperature is somewhat higher than for shot \#298069 and increases with time, in particular after the rf is shut off at $550 \mathrm{msec}$

\section{Summary and Discussion.}

The main aim of this paper is to document the PBXRAY code, which simulates the X-ray emission during lower hybrid current drive on PBX-M and creates computer generated images which are compared with the experimental images taken with the Hard X-ray Camera. The process of 
simulation was chosen as the first attempt to interpret the hard x-ray images (a) because it can be performed in an exact fashion, and (b) because of the complexity of the inversion. However, considerable progress has also been made recently in the inversion of the hard X-ray images.16,17 As far as we can tell, the only remaining uncertainty in the simulation is the variation of the density of suprathermal electrons on a magnetic flux surface, which was approximated in this paper with a $1 / \mathrm{R}$ dependence. This approximation seems satisfactory for most discharges, but can become a problem in discharges with a large number of trapped electrons.

The simulations quantitatively support the concept that images with a crescent-like X-ray intensity represent discharges where the suprathermal electrons created by the lower hybrid waves are located mostly on a radial shell. On PBX-M this has been one of the most encouraging findings for the attempt to modify the current profile with lower hybrid waves, in particular because the radial location of the shell changes for different phasings of the lower hybrid grill antenna. 18

Another encouraging finding is that the energy of the suprathermal electrons in the hollow discharges - as measured by the absorber foil method - is very low $\left(E \approx T_{\| f} \leq 100 \mathrm{keV}\right)$. The measured electron energy corresponds roughly to the phase velocity of the LH waves. The indications are that the hollow discharges occur in a regime where collisions dominate and rapidly transfer the momentum of the suprathermal electrons to the thermal distribution. The measured hot electron temperature again changes with phasing of the lower hybrid grill. 18 There is in some discharges a small hot electron component at higher energy near the plasma edge, which may be produced either by diffusion of very energetic electrons from the plasma center or by electron runaway in the low density edge region. However, the energetic component represents only 2 percent of the cool component and gives only a small contribution to plasma current. These results differ significantly from our earlier PLT measurements 1,7 and from data obtained on other machines $19,20,21,22$ where the hot electron energy $\left(T_{\| f}\right)$ is more in the 200 to $1000 \mathrm{keV}$ range.

Acknowledgments: The support of Drs. M. Okabayashi and N. Sauthoff and many helpful discussions with members of the PBX-M team are gratefully acknowledged. This work has been supported under Department of Energy contract No DE-AC02-76-CHO-3073. 


\section{References}

$\dagger$ Plasma Fusion Center, Massachusetts Institute of Technology, Cambridge, MA 02139.

$\dagger \dagger$ CRIP, Budapest, Hungary.

$\dagger_{\dagger \dagger}^{\dagger}$ JET, Joint Undertaking, Abingdon, Oxfordshire OX14 3EA, U.K..

1 J. Stevens, S. von Goeler, S. Bernabei, M. Bitter, T. K. Chu, et al.: Nucl. Fusion 25, p.1529 (1985)

2 R. Grimm, M. Chance, A. Todd, J. Manickam, M. Okabayashi, et al.: Nuclear Fusion 25, p.805 (1985)

3 N. Greenough, S. Bernabei, M. Norris, S. Schweitzer and R. Schwartz: Proc. 14th IEEE Symposium on Fusion Engineering, San Diego, Vol. 1, p.126 (1991)

4 S. von Goeler, J. Stevens, Ch. Karney, S. Bernabei, M. Bitter et al.: Proc 5th Topical APS Conf. on Radio Frequency Plasma Heating, Madison, p.96 (1983)

5 R. Kaita, S. von Goeler, S. Sesnic, S. Bernabei, E. Fredrickson, et al: Rev. Sci. Instrum. 61, p. 2756 (1990)

6 S. von Goeler, S. Bernabei, W. Davis, G. Gettelfinger, R. Kaita, et al.: Rev. Sci. Instrum. 65, p. 1621 (1994)

7 S. von Goeler, J. Stevens, S. Bernabei, M. Bitter, T. K. Chu, et al.: Nucl. Fusion 25, p.1515 (1985)

8 C. Karney and N. Fisch: Phys. Fluids 22, p.1817 (1985)

9 N. Fisch, Rev. Mod. Phys. 59, p.175 (1987)

10 D. Ignat, E. Valeo, and S. von Goeler: Proc. 10th Topical Conf. on Radio Freq. Power in Plasmas (Boston), AIP Conf. Proc. 289, p.127 (1993).

11 R. Gluckstern and M. Hull: Phys. Rev. 90, p.1030 (1953)

12 G. Elwert: Ann. Physik 34, p.178 (1939)

13 E. Haug: Z. Naturforsch. 30, p 1099 (1975)

14 S. von Goeler, W. Stodiek, H. Eubank, H. Fishman, S. Grebenshchikov, et al.: Nuclear Fusion 15, p. 301 (1975)

15 W. McMaster, N. Kerr Del Grande, J. H. Mallett, and J. H. Hubbell: Compilation of X-ray Cross Sections, Lawrence Livermore Radiation Laboratory Report UCRL-50174 (1969).

16 S. Jones, "Fast Electron Transport and Lower Hybrid Absorbed Power Profiles from Hard X-ray Imaging on PBX-M", Bull. APS 38, p.1985, (1993), to be published Phys. Plasmas.

17 R. Bell: Proc. 10 th Topical Conf on High-Temperature Plasma Diagnostics, Rochester, to be published Rev. Sci. Instrum (1994).

18 S. von Goeler, S. Jones, S. Bernabei, D. Ignat, R. Kaita et al.: Bull. APS 38, p.2093, (1993), to be published.

19 S. Texter, S. Knowlton, M. Porkolab, Y. Takase: Nucl. Fusion 26, p.1279 (1986) 
20 M. Brusati, D. Bartlett, A. Ekedahl, P. Froissard, A. Airoldi, et al.: JET laboratory report JET-P(93)54, (1993) to be published Nuclear Fusion.

21 J. P. Bizarro, Y. Peysson, P. Bonoli, J. Carasco, T. Dudok de Wit, et al.: Proc. 20th EPS Conf. Contr. Fusion Plasma Physics, Lisboa, Vol.III, p. 889, (1993)

22 Y. Peysson: Plasma Phys. Control. Fusion 35, B253 (1993) 


\section{Figure Captions:}

Fig. 1: Mechanical Layout of the Hard X-ray Camera. Hard X-ray bremsstrahlung, which is emitted from the plasma volume, enters the pinhole camera through an interchangeable aperture. It is detected by a medical X-ray image intensifier, which converts the X-ray image into a visible image. The visible image is recorded, after amplification, by a self-scanning RETICON diode array. Low energy $\mathrm{X}$-ray photons can be attenuated by an absorber foil.

Fig. 2: Typical image recorded by the Hard X-ray Camera exhibiting a hollow X-ray profile.

Fig. 3: Anisotropy of the hard X-ray emission. Shown are bremsstrahlung emission cones for relativistic electrons, going from left to right. Parameter of the curves is the energy of the emitted X-ray photons. Consider a vector from the origin to a point on one of the curves. Its length is proportional to the X-ray intensity emitted in the direction of the vector at the photon energy associated with the particular curve.

Fig. 4: Electron momentum distribution functions used for the simulation of PBX-M shot \#298069. The dominant low-energy component with $\left(\mathrm{T}_{\| \mathrm{f}}=100 \mathrm{keV}, \mathrm{T}_{\perp}=\mathrm{T}_{\| \mathrm{b}}=10 \mathrm{keV}, \mathrm{E}^{*}=500 \mathrm{keV}\right)$ is shown in (a). The high plateau in the center with steep slopes on the sides indicates the thermal distribution $\left(\mathrm{T}_{\mathrm{th}}=1 \mathrm{keV}\right)$. A small high-energy component $\left(\mathrm{T}_{\| \mathrm{f}}=400 \mathrm{keV}, \mathrm{T}_{\perp}=\mathrm{T}_{\| \mathrm{b}}=50 \mathrm{keV}, \mathrm{E}^{*}=999 \mathrm{keV}\right)$, which is found at the plasma edge, is shown in (b).

Fig. 5: Bean shaped plasma equilibrium calculated by the FQ code for shot \#298069.

Fig. 6: Radial profile of the number density of suprathermal electrons used to simulate shot \#298069. The contributions by the central low energy component $\left(\mathrm{T}_{\| \mathrm{f}}=100 \mathrm{keV}, \mathrm{T}_{\perp}=\mathrm{T}_{\| \mathrm{b}}=10 \mathrm{keV}\right)$ and the outer high energy component $\left(\mathrm{T}_{\| \mathrm{f}}=400 \mathrm{keV}, \mathrm{T}_{\perp}=\mathrm{T}_{\| \mathrm{b}}=50 \mathrm{keV}\right.$ ) (compare Fig. 4) are indicated by the dotted and the dashed curves, respectively.

Fig. 7: Images for electron rings centered at different flux surfaces $\Psi_{\text {se. }}$ ((a) $\Psi_{\text {se }}=0.00$, (b) $\Psi_{\text {se }}=0.25$, (c) $\Psi_{\text {se }}=0.50$, and (d) $\Psi_{\text {se }}=0.75$ ). The fall-off distances for the suprathermal electron density were all 
set to $\Delta \Psi_{\text {seo }}=\Delta \Psi_{\text {sei }}=0.20$. The equilibrium file used in the computation is shown in Fig. 5, the hot electron temperatures were $\mathrm{T}_{\| \mathrm{f}}=100 \mathrm{keV}, \mathrm{T}_{\perp}=10 \mathrm{keV}, \mathrm{T}_{\| \mathrm{b}}=10 \mathrm{keV}$ and $\mathrm{E}^{*}=500 \mathrm{keV}$.

Fig. 8: Dependence of the maximum X-ray intensity of the image on the suprathermal electron temperature $T_{\| f} .\left(T_{\perp}=T_{\| b}=0.25 T_{\| f}\right)$. The X-ray intensity is normalized to the intensity at $\mathrm{T}_{\| \mathrm{f}}=400$ $\mathrm{keV}$ for the no-foil case.

Fig. 9: Dependence of the horizontal profile shape on electron energy. Parameter of the curves is the parallel forward temperature $T_{\| f}$. $\left(T_{\perp}=T_{\| b}=0.25 T_{\| f}\right)$. The maximum intensity of the image is normalized to unity.

Fig. 10: Dependence of the horizontal profile shape on isotropy. Parameter of the curves is the perpendicular temperature, $T_{\perp}=T_{\| b}$; the parallel forward temperature was kept fixed, $T_{\| f}=400 \mathrm{keV}$. The maximum intensity of the image is normalized to unity.

Fig. 11: q value at tangency radius of hard X-ray sight lines for a circular plasma.

Fig. 12: Image for a very energetic anisotropic distribution with $\mathrm{T}_{\| \mathrm{f}}=999$ $\mathrm{keV}, \mathrm{T}_{\perp}=50 \mathrm{keV}, \mathrm{T}_{\| \mathrm{b}}=50 \mathrm{keV}$ and $\mathrm{E}^{*}=999 \mathrm{keV}$ for a circular plasma (Equilibrium file for shot $\# 291430, \Psi_{\text {se }}=0.5, \Delta \Psi_{\text {seo }}=\Delta \Psi_{\text {sei }}$ $=0.30$ )

Fig. 13: Plasma current $I_{p}$, plasma line density $n_{e} l$, lower hybrid power $\mathrm{P}_{\mathrm{LH}}$, and a central and an outer soft X-ray trace versus time for plasma shot \#291430.

Fig. 14: (a) Contour plot of the X-ray intensity for shot \#291430 averaged for time 390 to $410 \mathrm{~ms}$. A Cu foil is inserted in the X-ray path. (b) Simulation of shot 291430 (The suprathermal electron density profile that served as input is shown in Fig. 16). Contour levels are at $0.12,0.16,0.23,0.32,0.44,0.62$, and 0.87 of the maximum of the image.

Fig. 15: Horizontal and vertical profiles for shot \#291430. Solid curves are from the experiment, dashed curves are simulated. 
Fig. 16: Density of suprathermal electrons $n_{\text {se }}(R)$ used for the simulations of shot \#291430 shown in Fig. 13 and 14. The total density (solid curve) is the sum of two Gaussians (the dotted and the dashed curve). Both Gaussians have the same momentum distribution [ $\mathrm{T}_{\| \mathrm{f}}$ $=200 \mathrm{keV}, \mathrm{T}_{\perp}=50 \mathrm{keV}, \mathrm{T}_{\| \mathrm{b}}=50 \mathrm{keV}$ and $\left.\mathrm{E}^{*}=500 \mathrm{keV}\right]$.

Fig. 17: Vertical profiles of the hard X-ray emission vs. time for shot \#291430.

Fig. 18:Discharge conditions for the hollow discharge shot \#298069. Plasma current $I_{p}$, plasma line density $n_{e} 1$, lower hybrid power $\mathrm{P}_{\mathrm{LH}}$, neutral beam heating power $\mathrm{P}_{\mathrm{NB}}$, and soft X-ray traces vs. time. A modest amount of neutral beam injection $\mathrm{P}_{\mathrm{NB}}$ often proved useful to stabilize MHD instabilities caused by the lower hybrid current drive.

Fig. 19: (a) Contour plot of the X-ray intensity for shot \#298069 averaged for time 270 to $295 \mathrm{~ms}$. No foil was inserted in the X-ray path. (b) Simulation of shot 298069, using the suprathermal electron density profile shown in Fig. 6.

Fig. 20: Horizontal and vertical profiles for shot \#298069. Solid curves are from the experiment, dashed curves are simulated.

Fig. 21: Vertical profiles vs. time for shot \#298069.

Fig. 22: (a) Contour plot of the photon temperature determined from shot \#298069 (no foil) and shot \#298070 (Cu foil) averaged from time 270 to $295 \mathrm{~ms}$. (b) Simulation of photon temperature.

Fig. 23: (a) Horizontal profiles of the photon temperature. Solid curve from shot \#298069 (no foil) and \#298070 (Cu foil), dashed dotted curve from shot \#298069 (no foil) and \#298071 (Mo foil). (b) Simulation of the photon temperature for shot \#298069.

Fig. 24: Simulation of horizontal profiles of the photon temperature for shot \#298069 using different values for the hot electron temperatures $T_{\| f}, T_{\perp}$, and $T_{\| b}$.

Fig. 25: Central photon temperature vs. time for shot \#298069.

Fig. 26: Central photon temperature vs. time for shot \#291430. 


\section{HARD X-RAY CAMERA ON PBX-M}

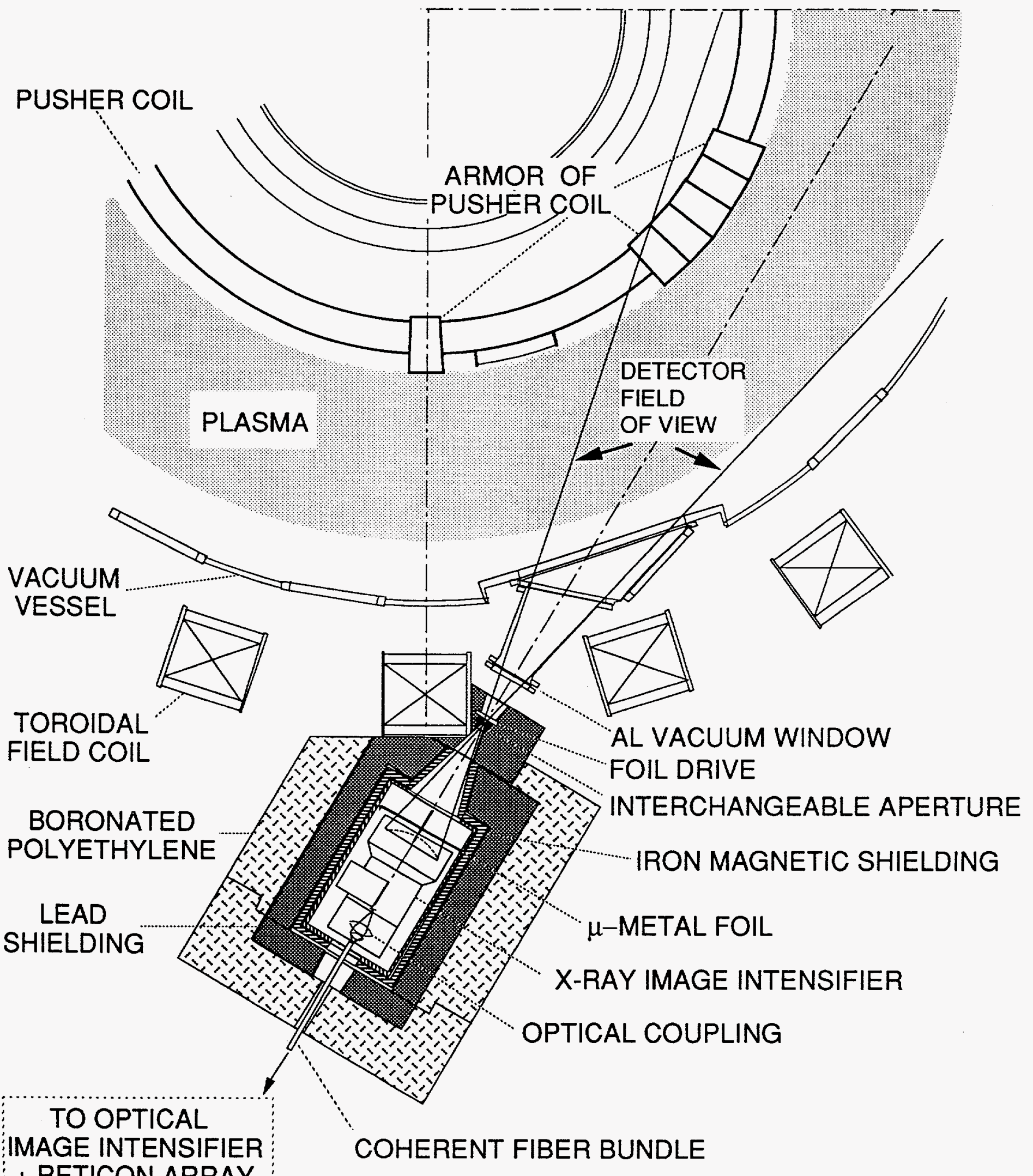


Shot 298069: Frames 6-8

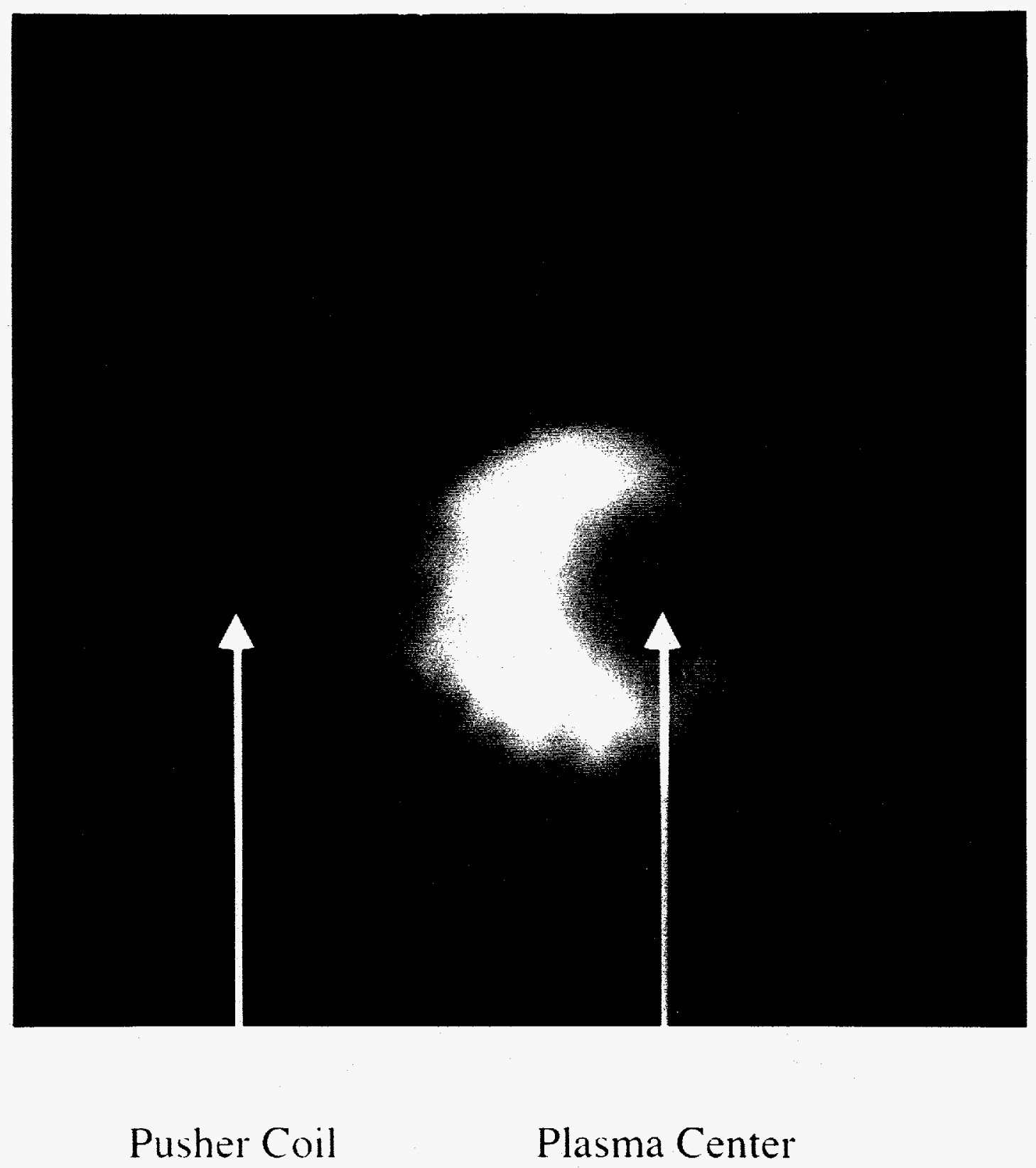

Fig. 2 


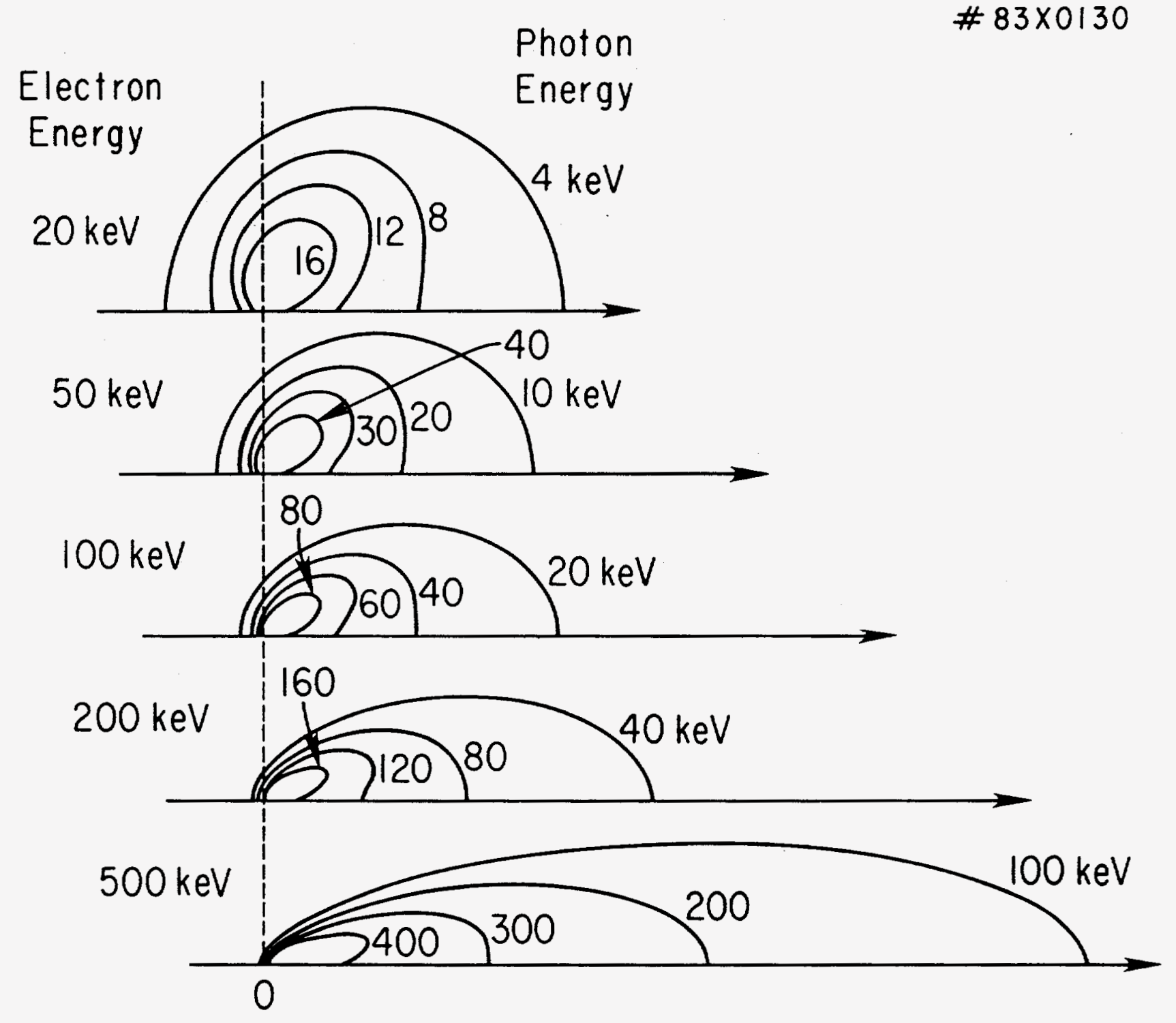

Fig. 3 

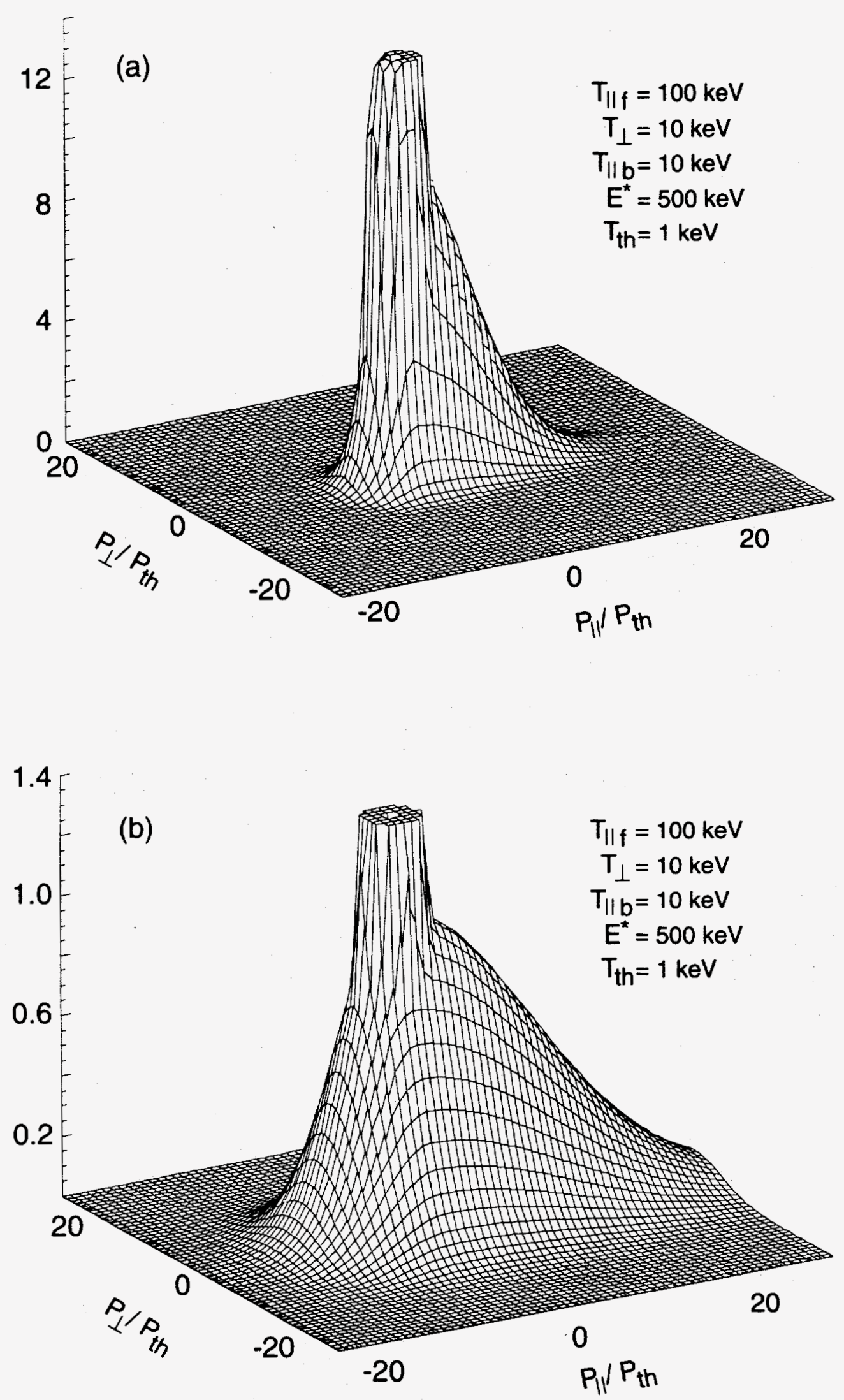

Fig. 4 


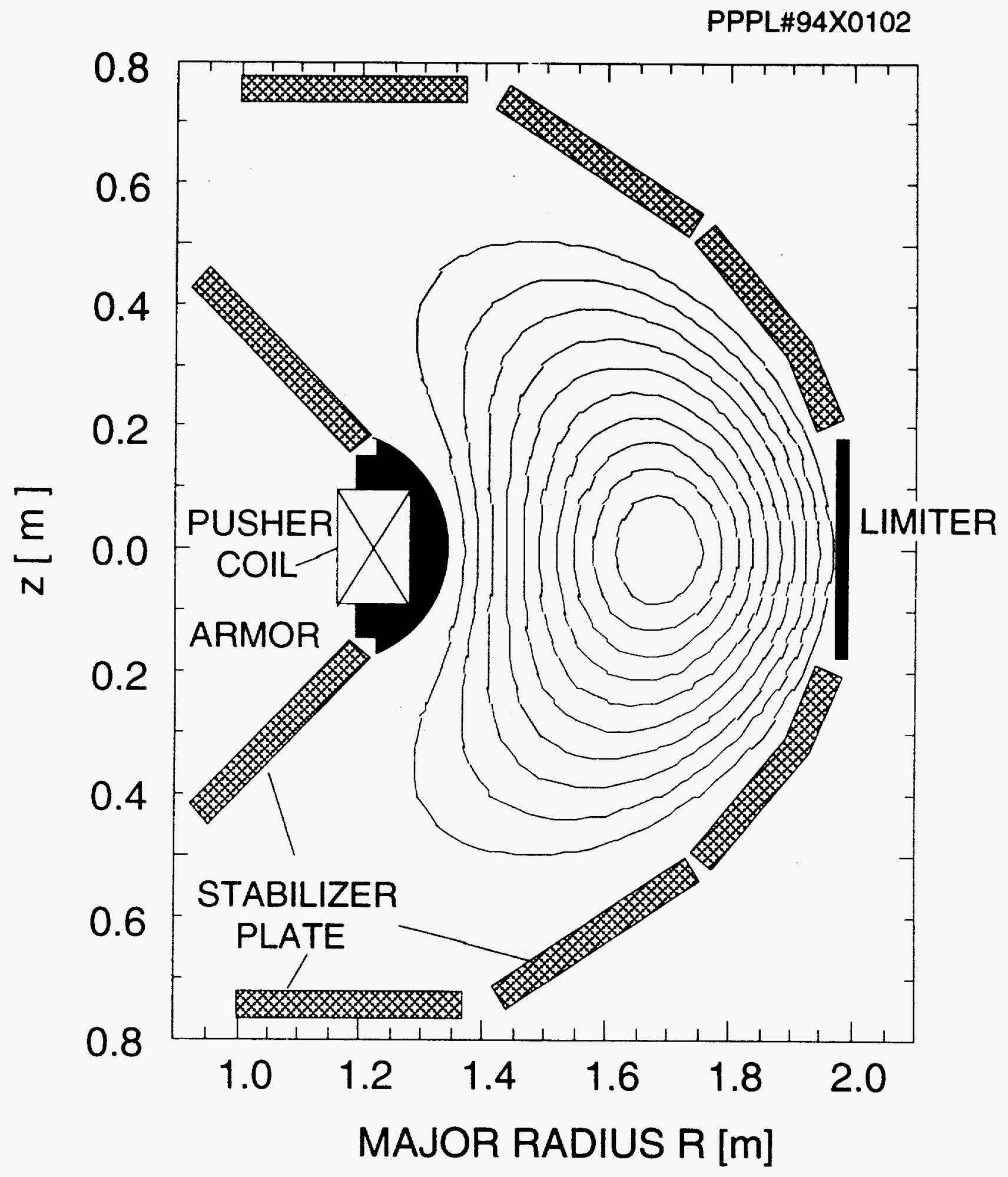

Fig. 5 


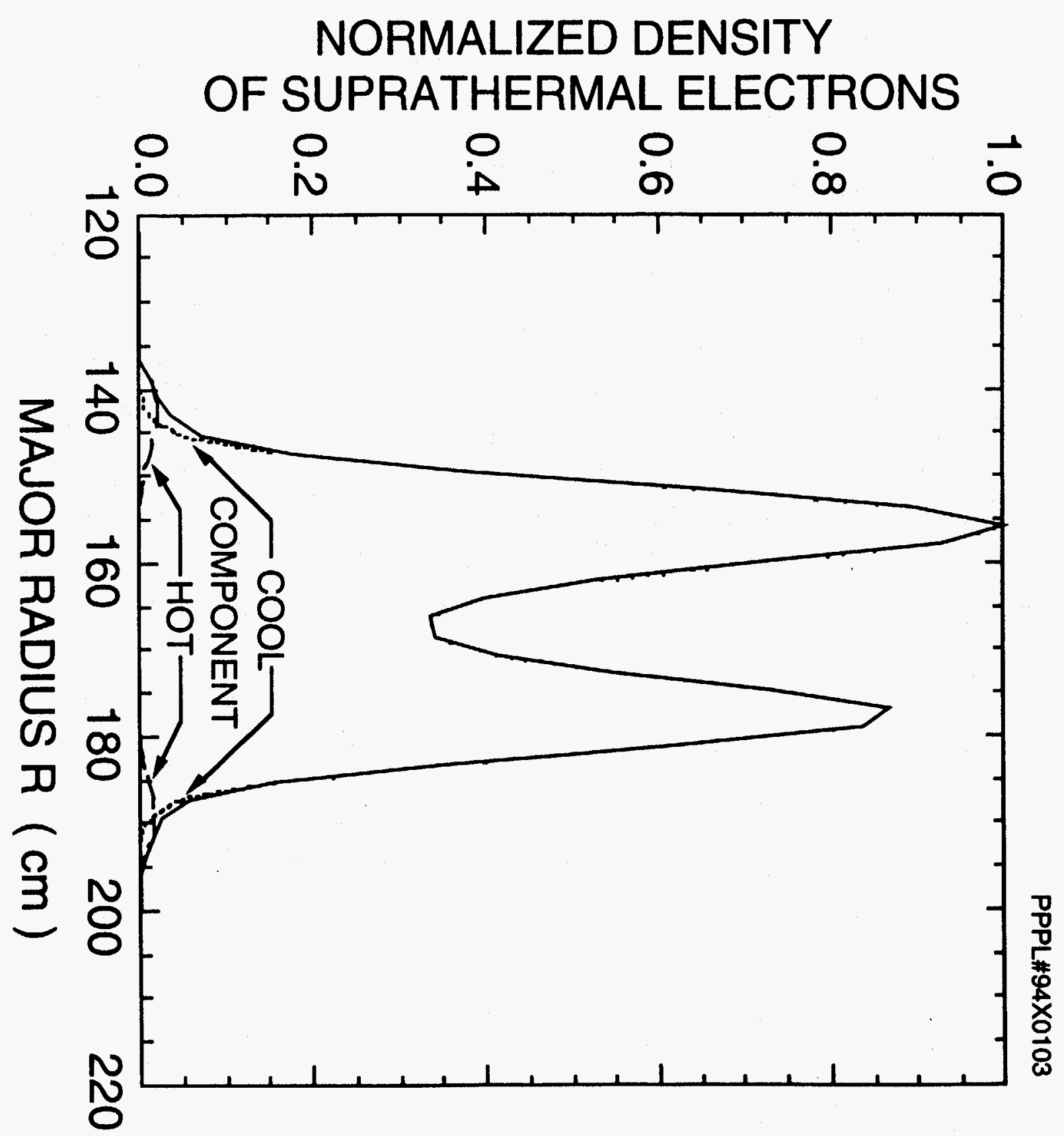


PPPL $\$ 94 \times 0104$
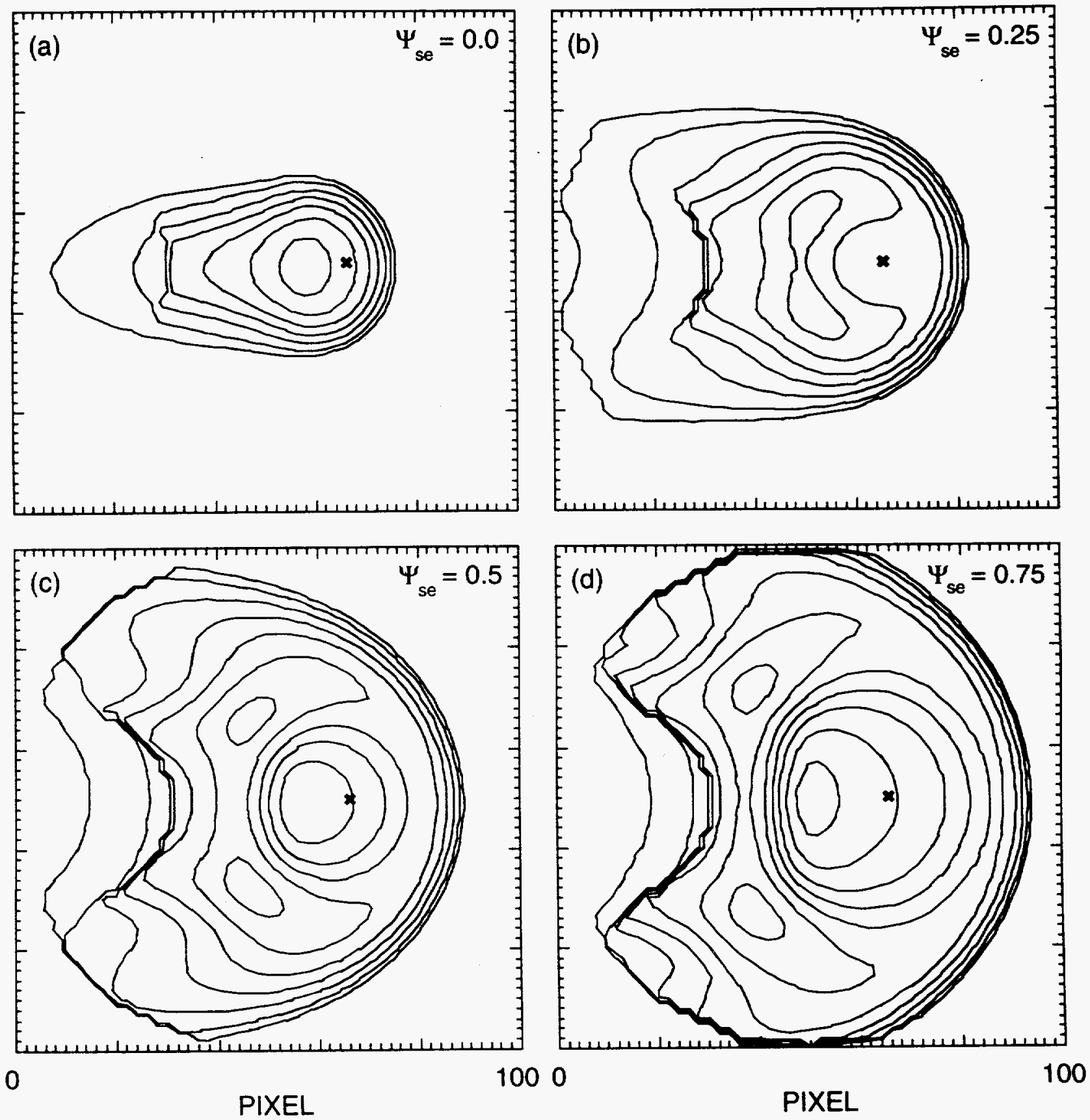
$8 \cdot 8$ ำ

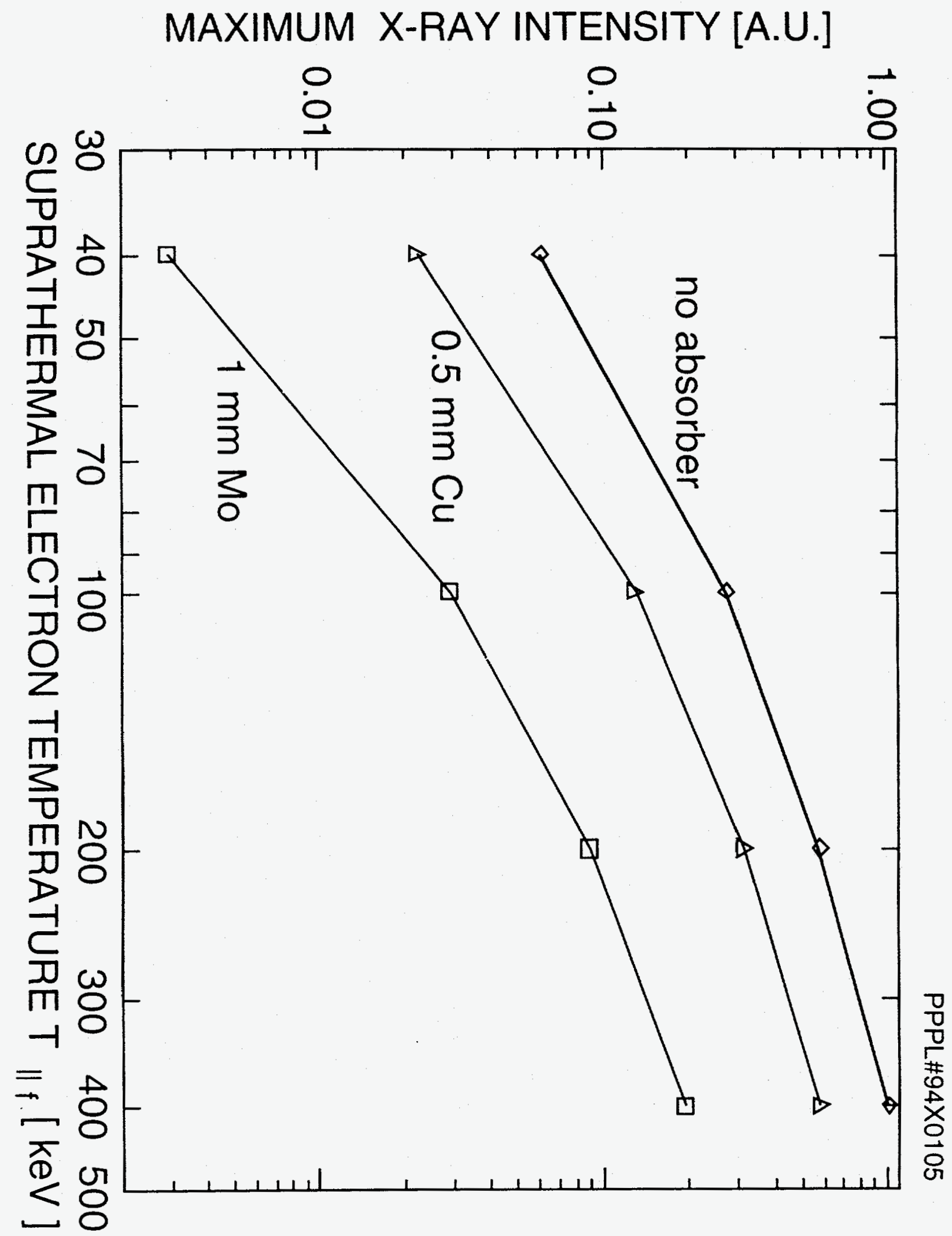


PPPL\#94X0106

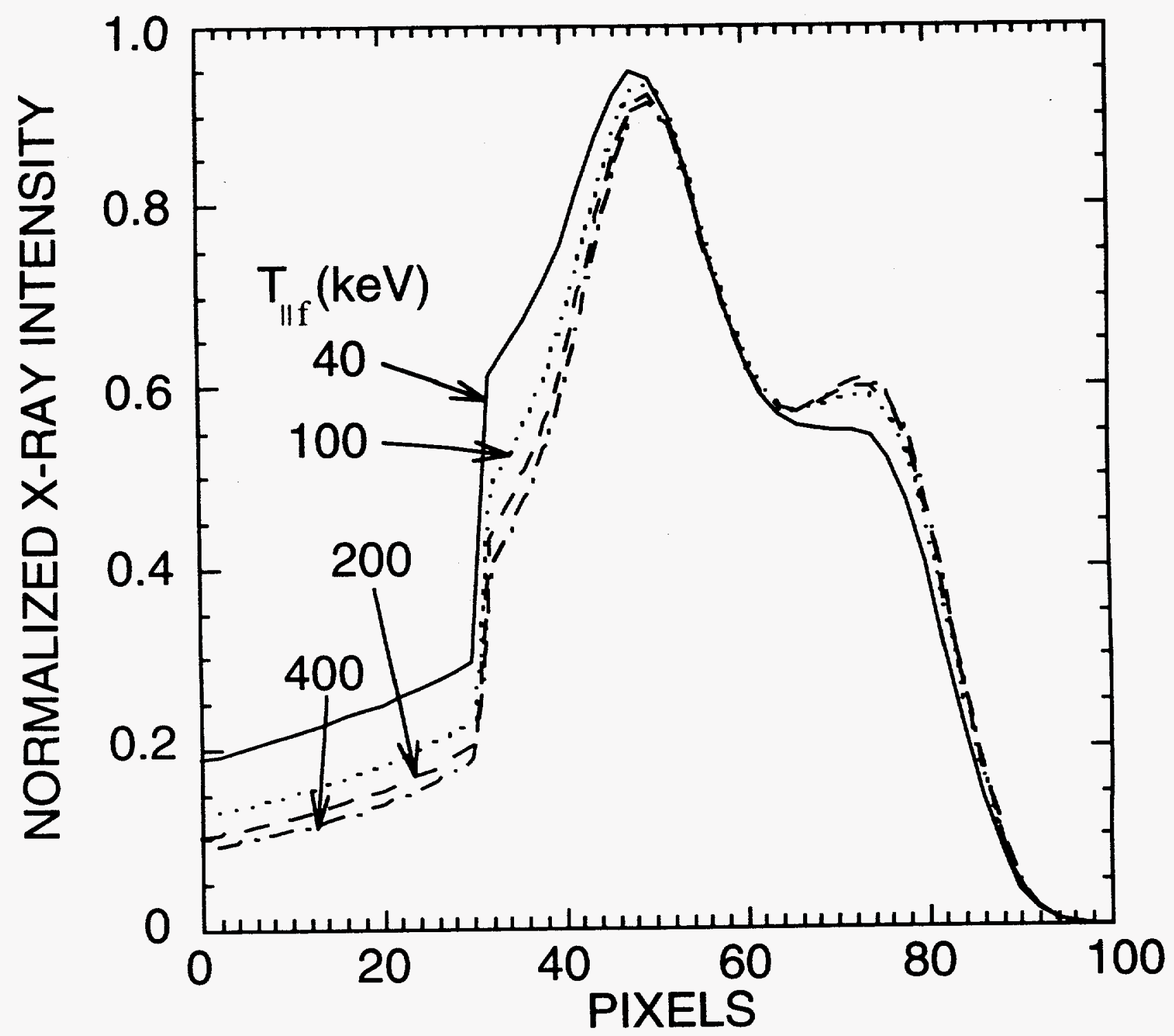

Fig。9 
PPPL\#94X0107

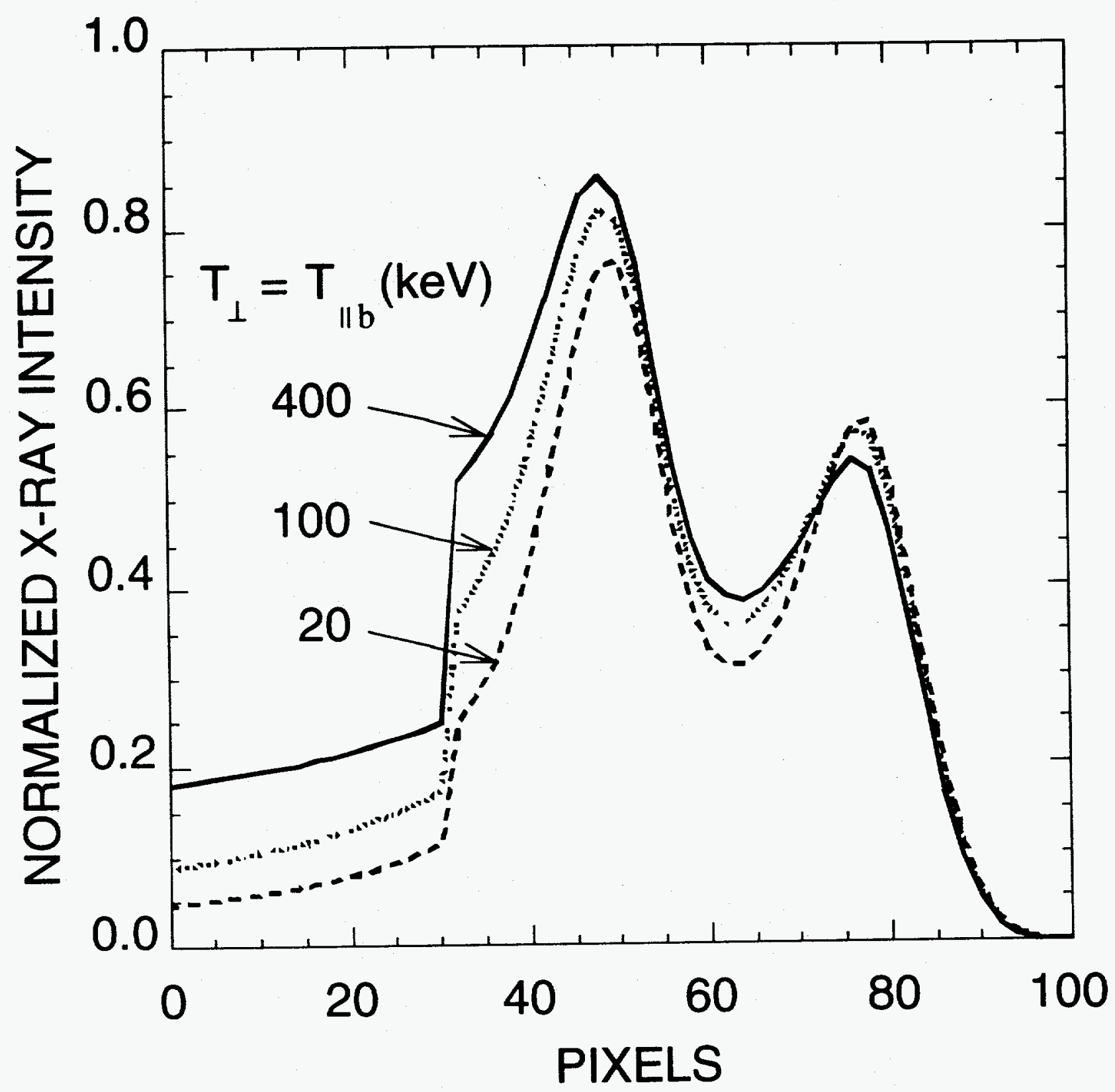

Fig. 10 
PPPL\#94X0108

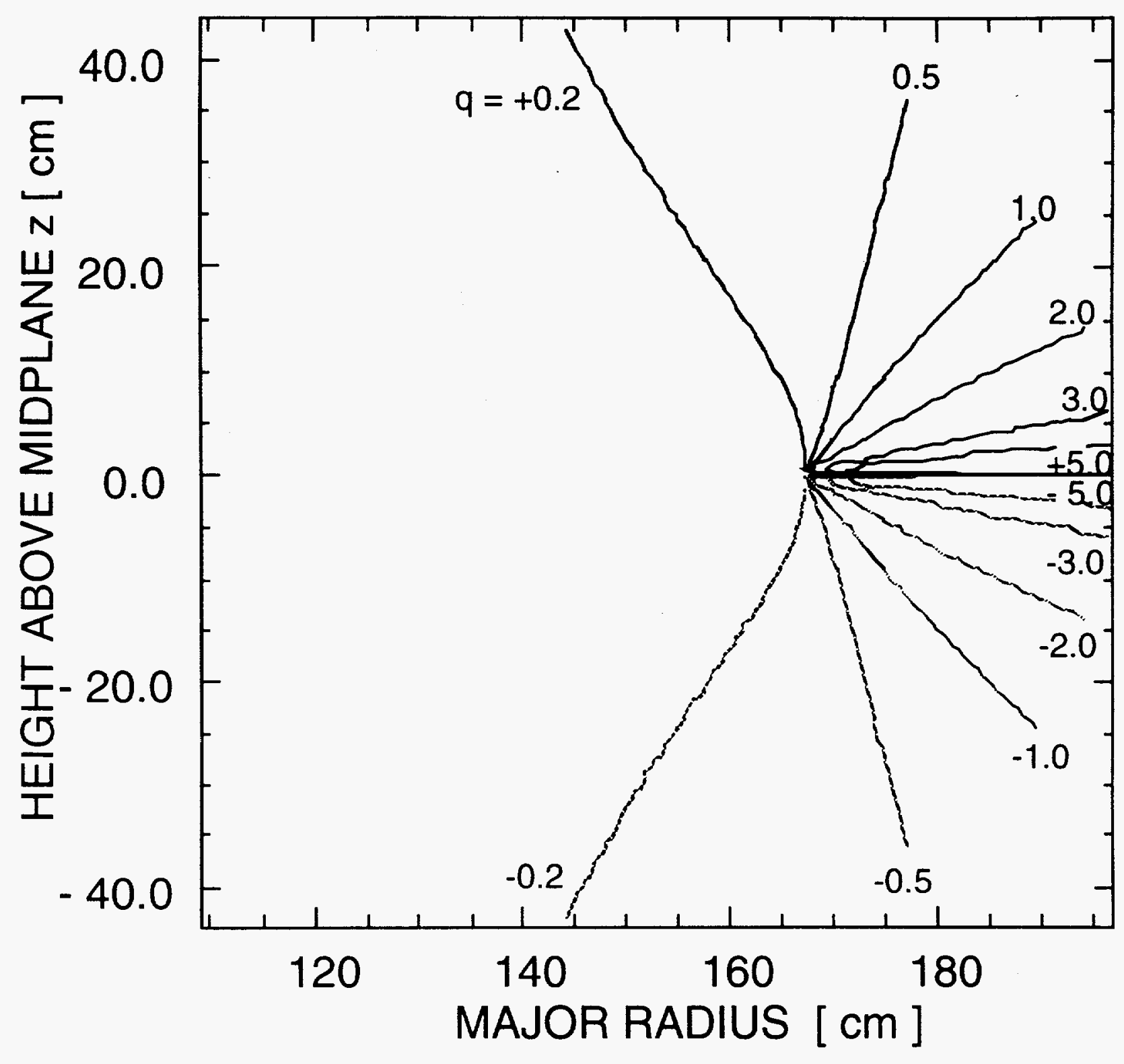




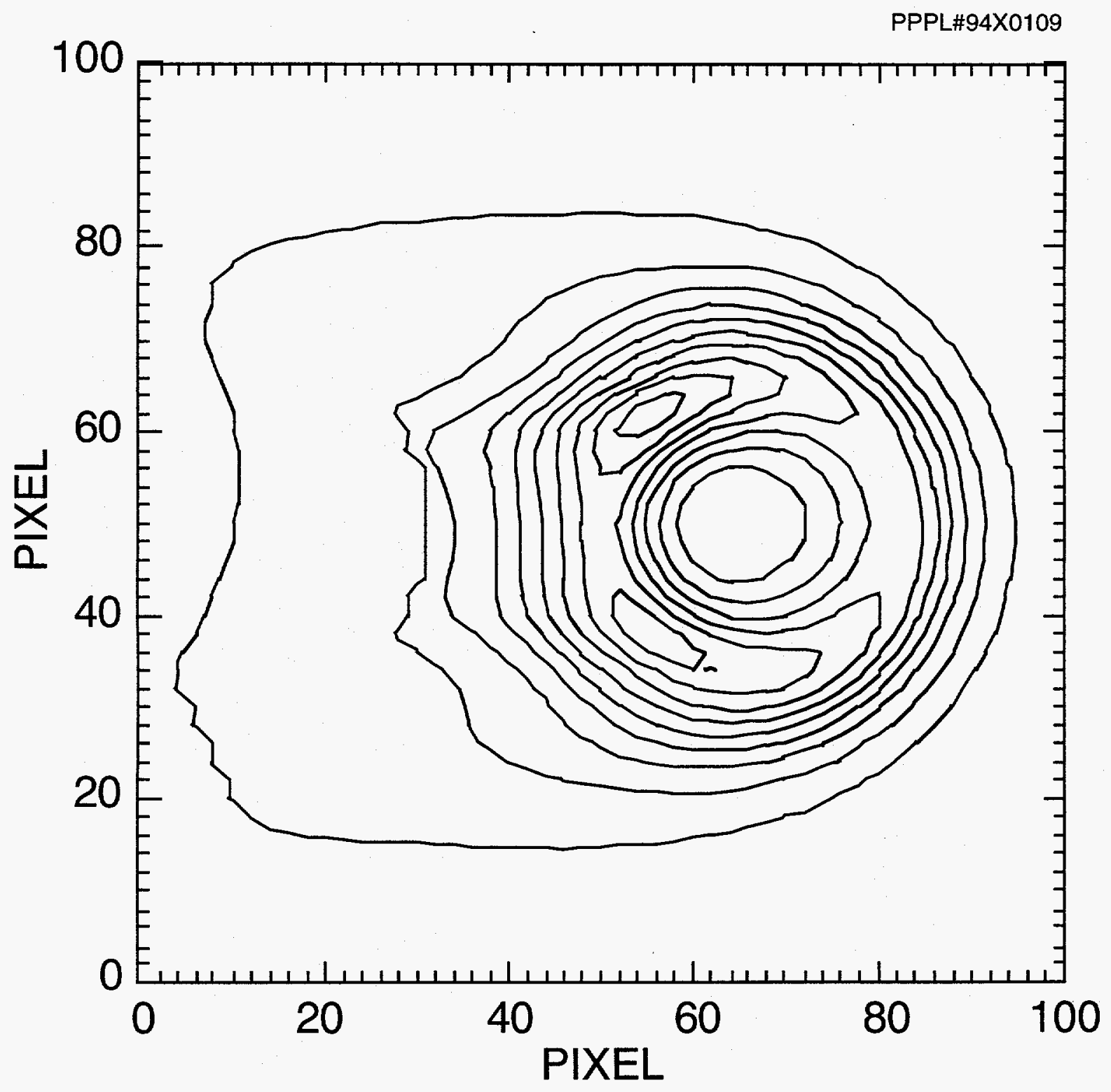

Fig. 12 
PPPL\#94X0110

SHOT \#291430

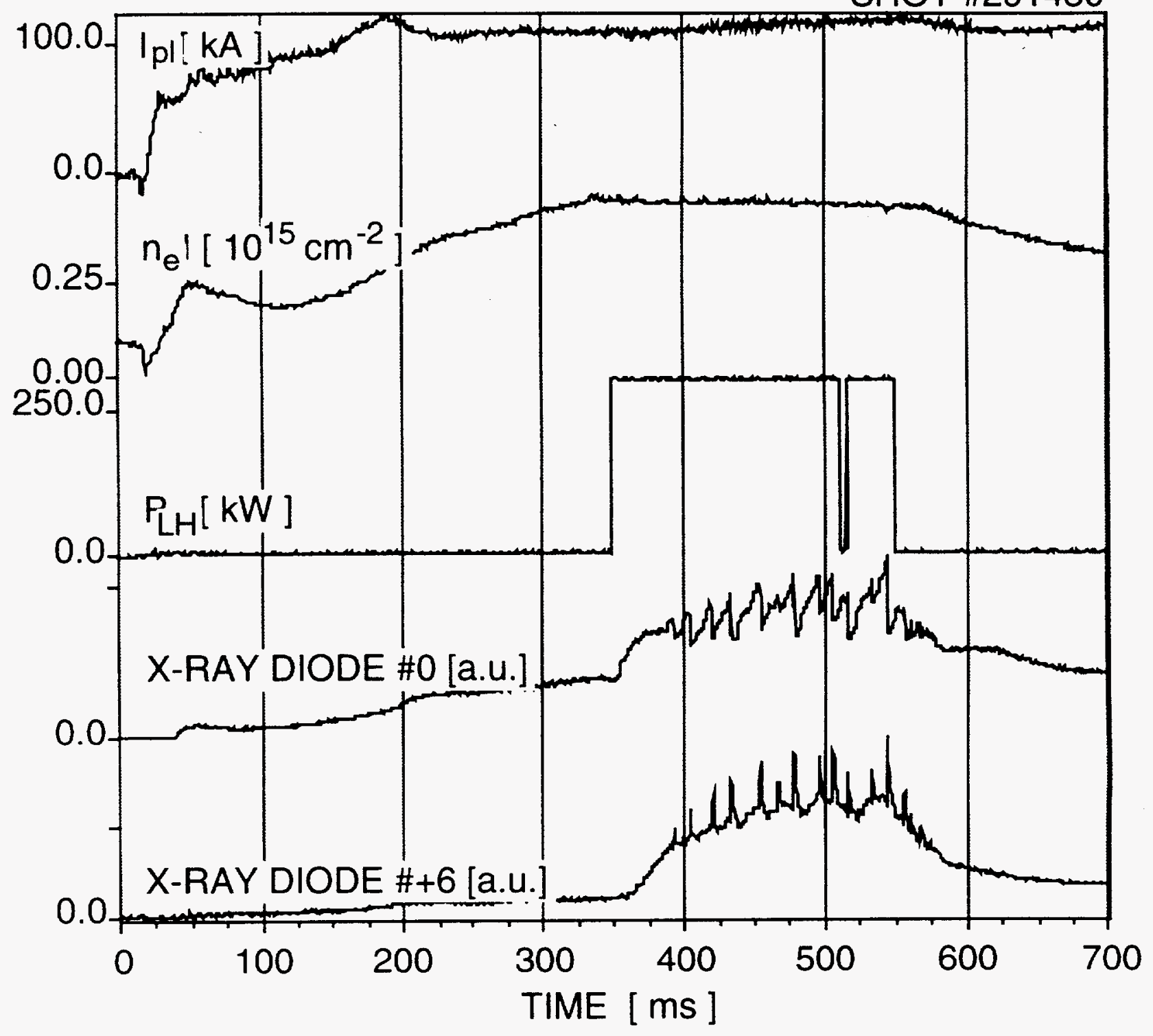




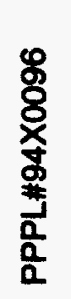

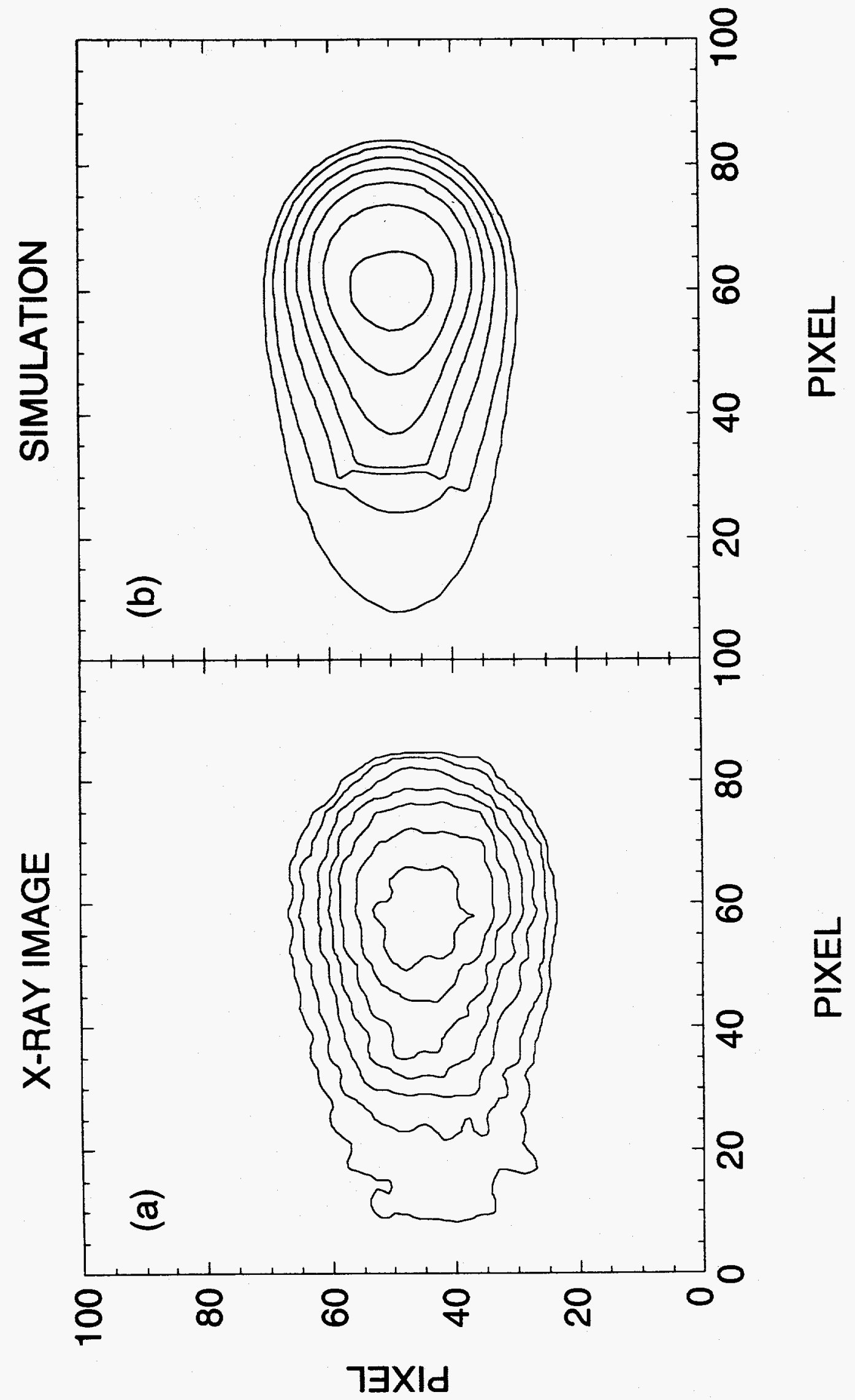




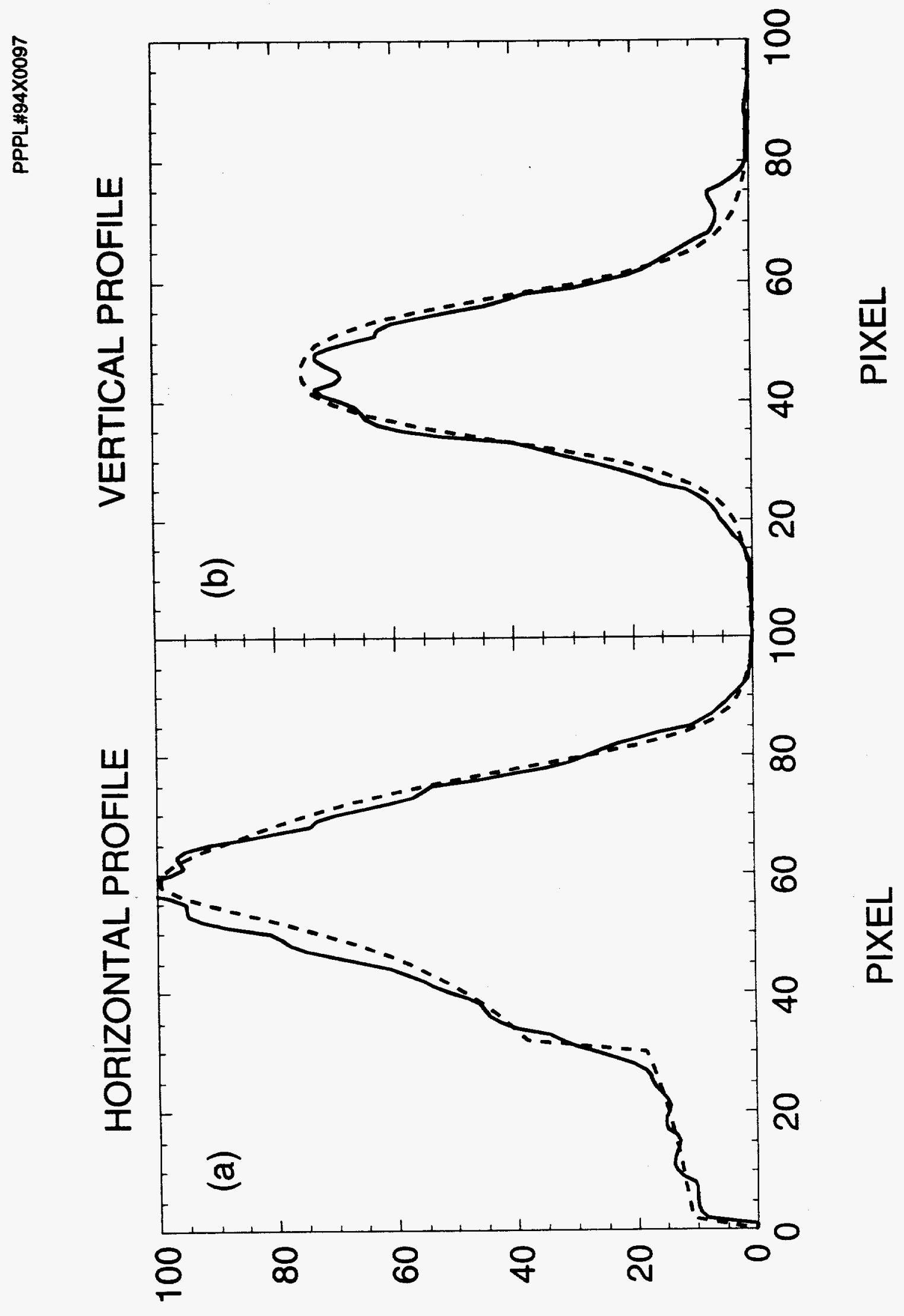

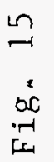


PPPL\#94X0098

\section{DENSITY OF SUPRATHERMAL ELECTRONS}

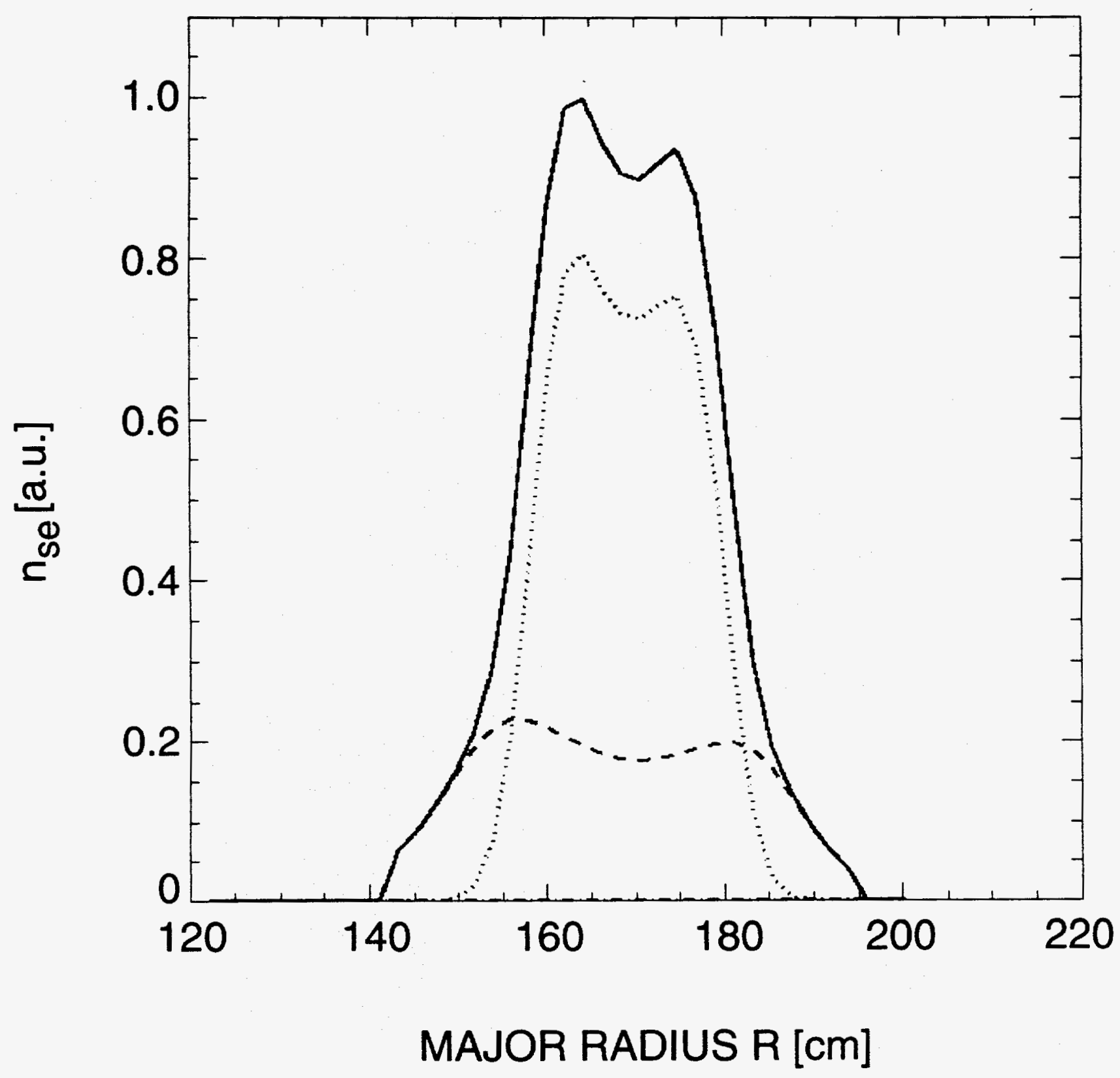

Fig. 16 


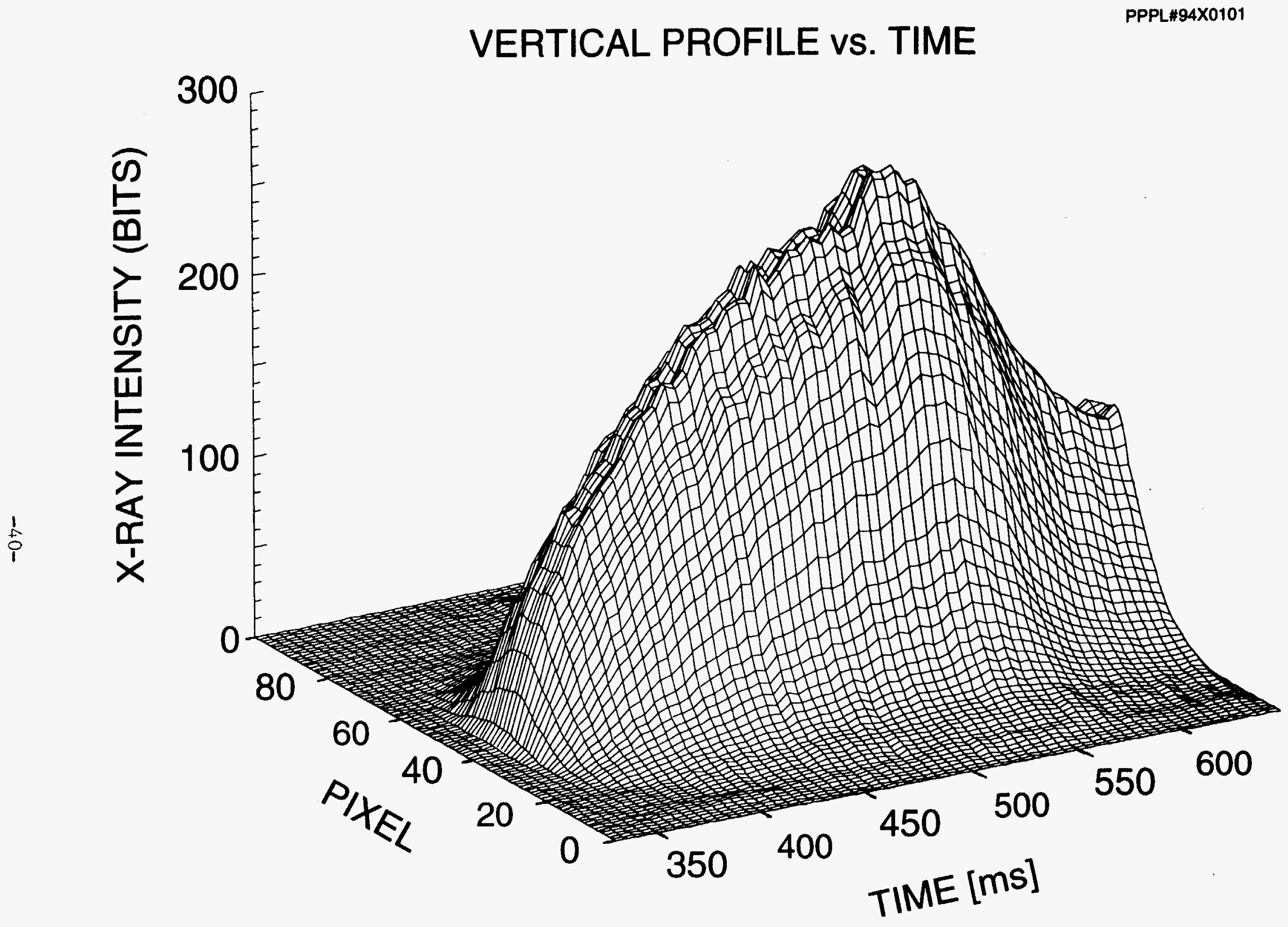


PPPL\#94X0111

SHOT \#298069

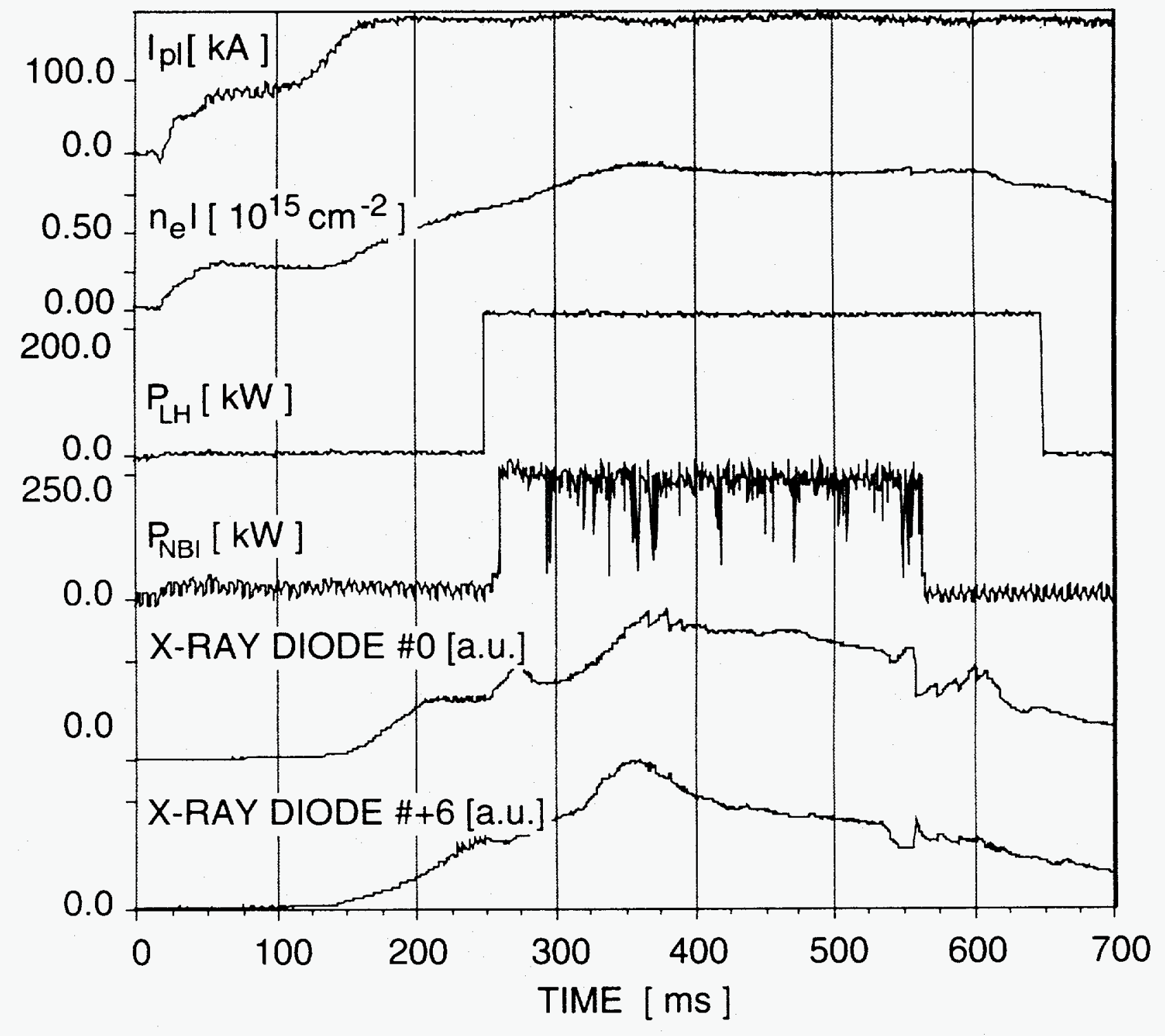

Fig. 18 


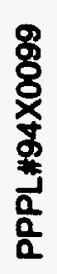

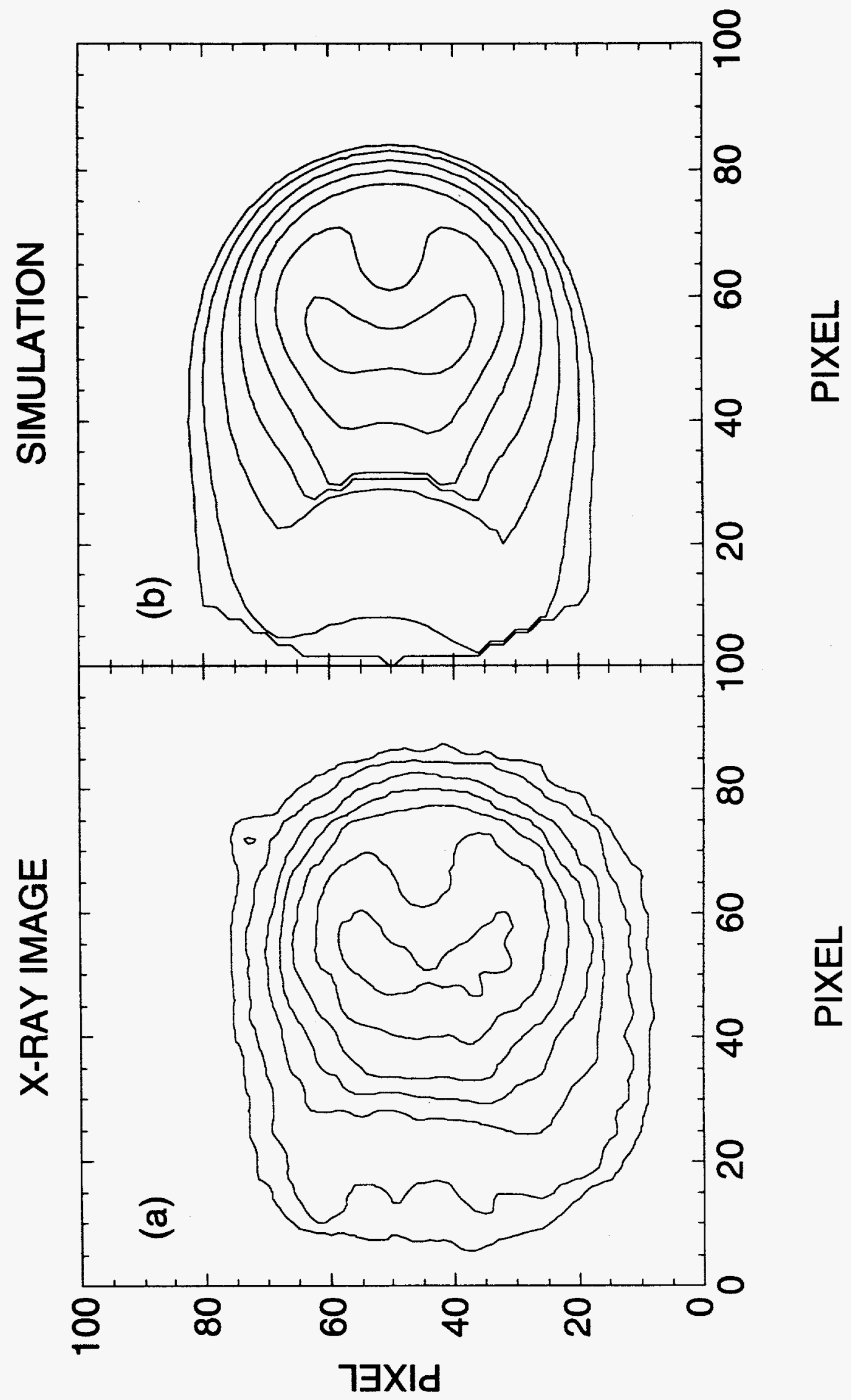

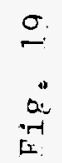




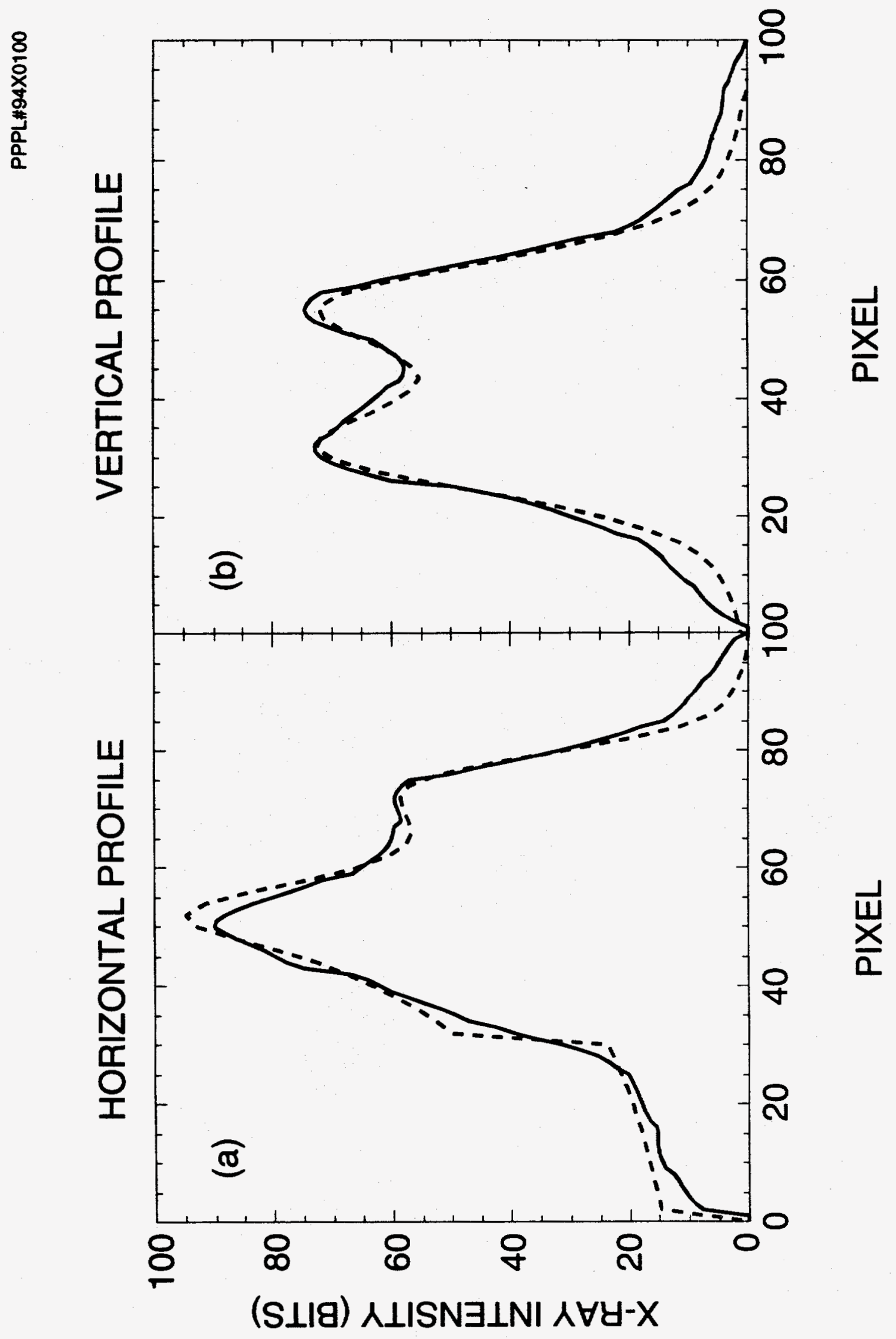


$\frac{9}{0}$
웅
$\frac{0}{0}$
0
0
0
0

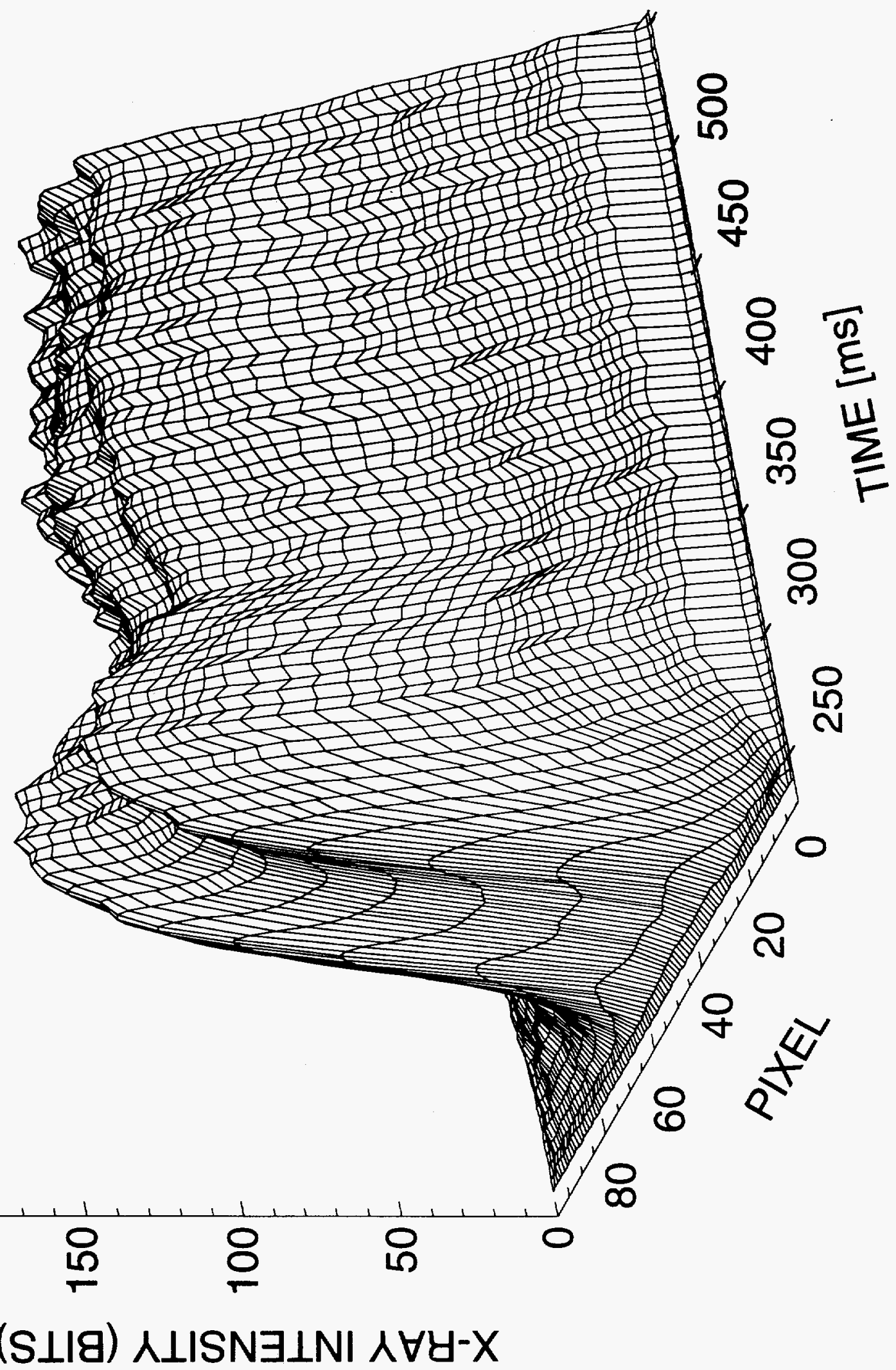



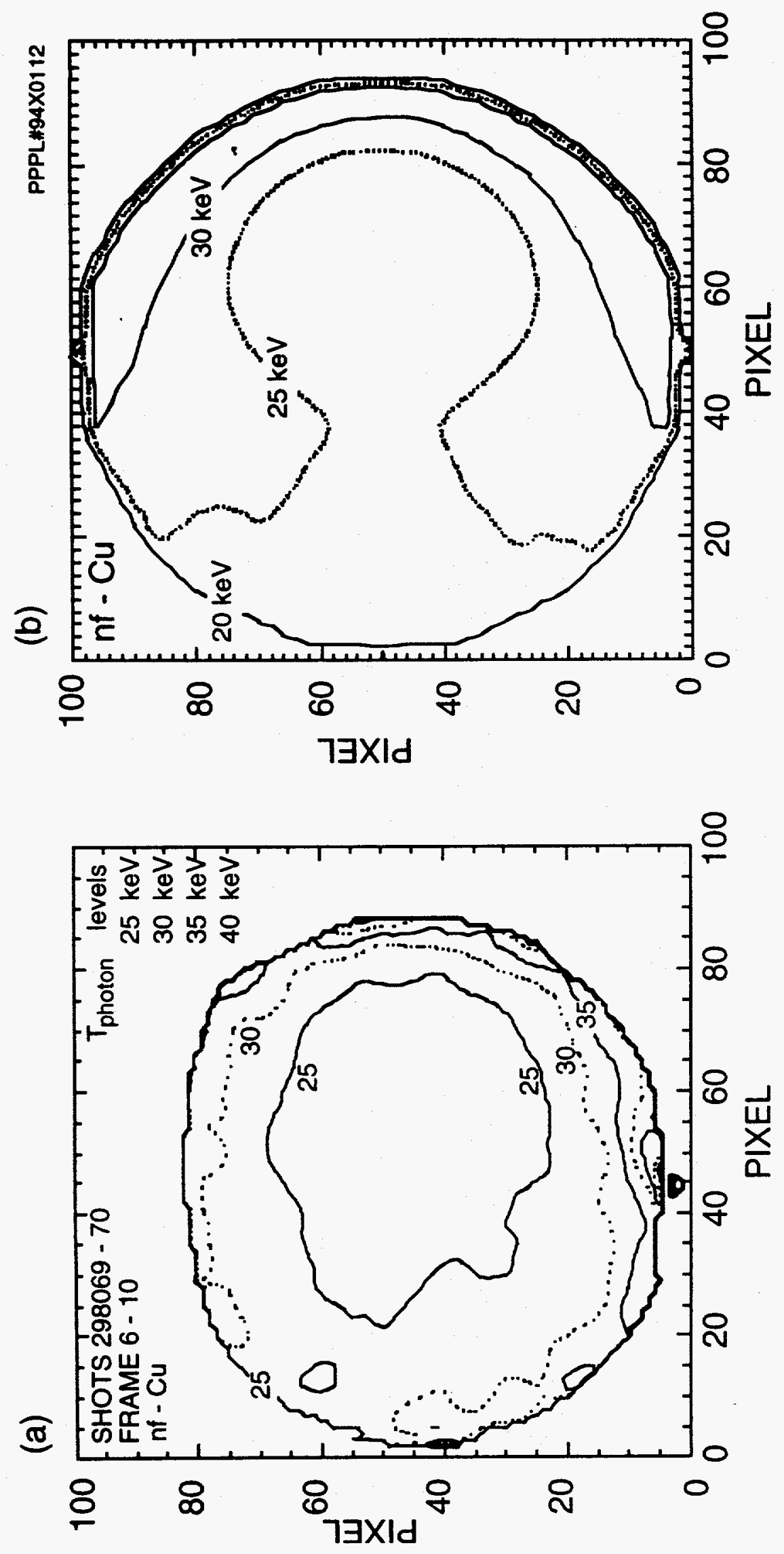
PPPL\#94X0114
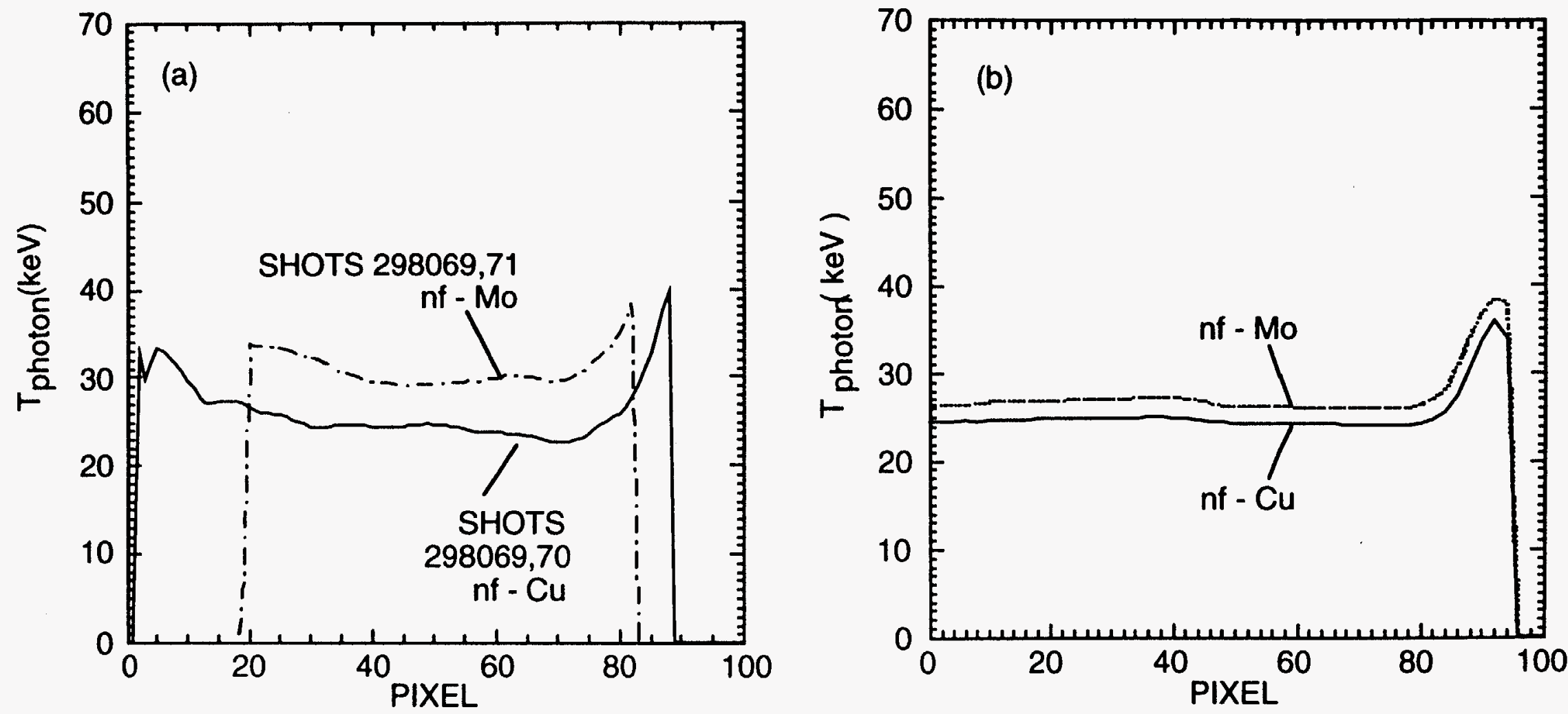
PPPL\#94X0116

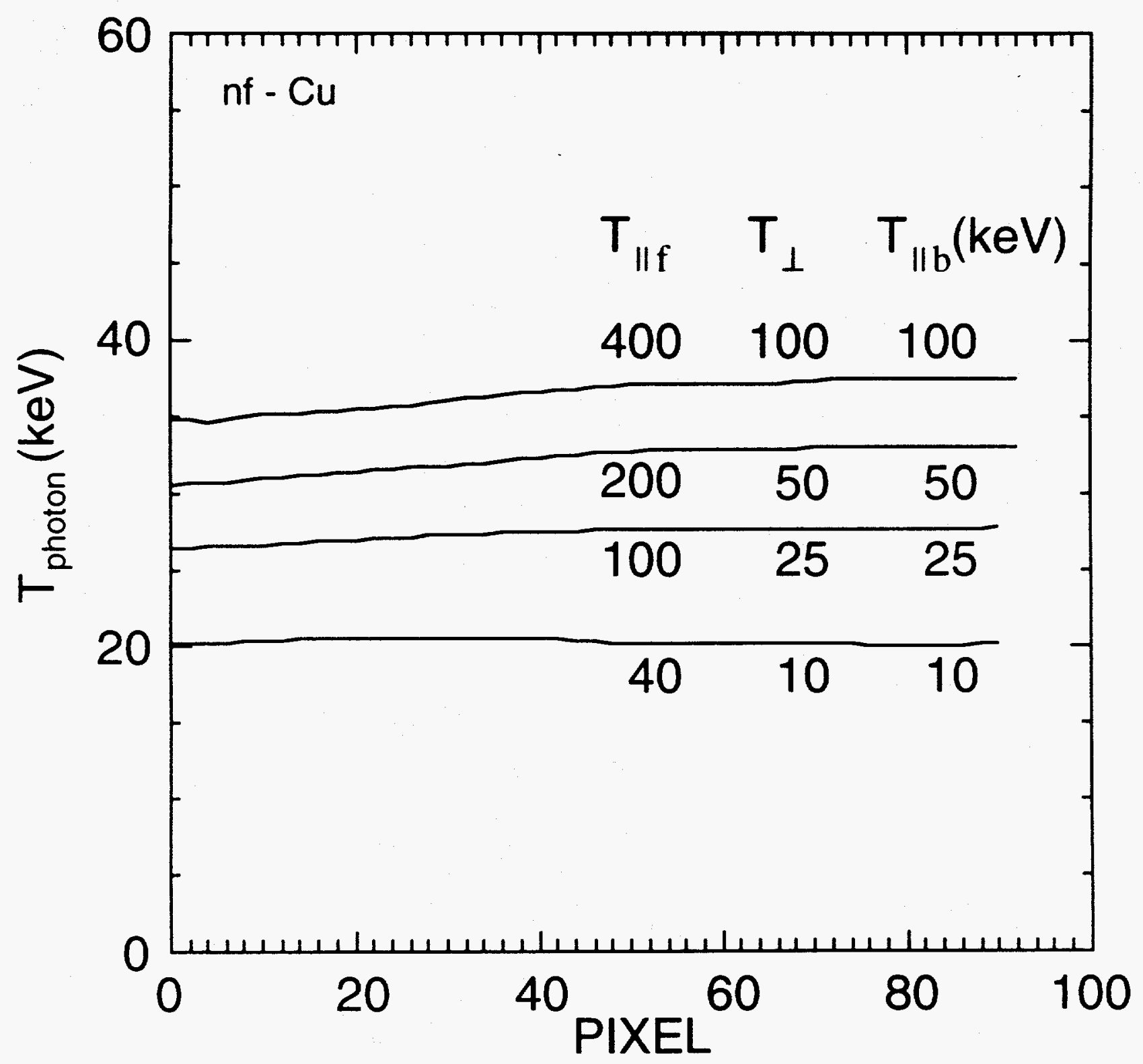

Fig. 24 
PPPL\#94X0117

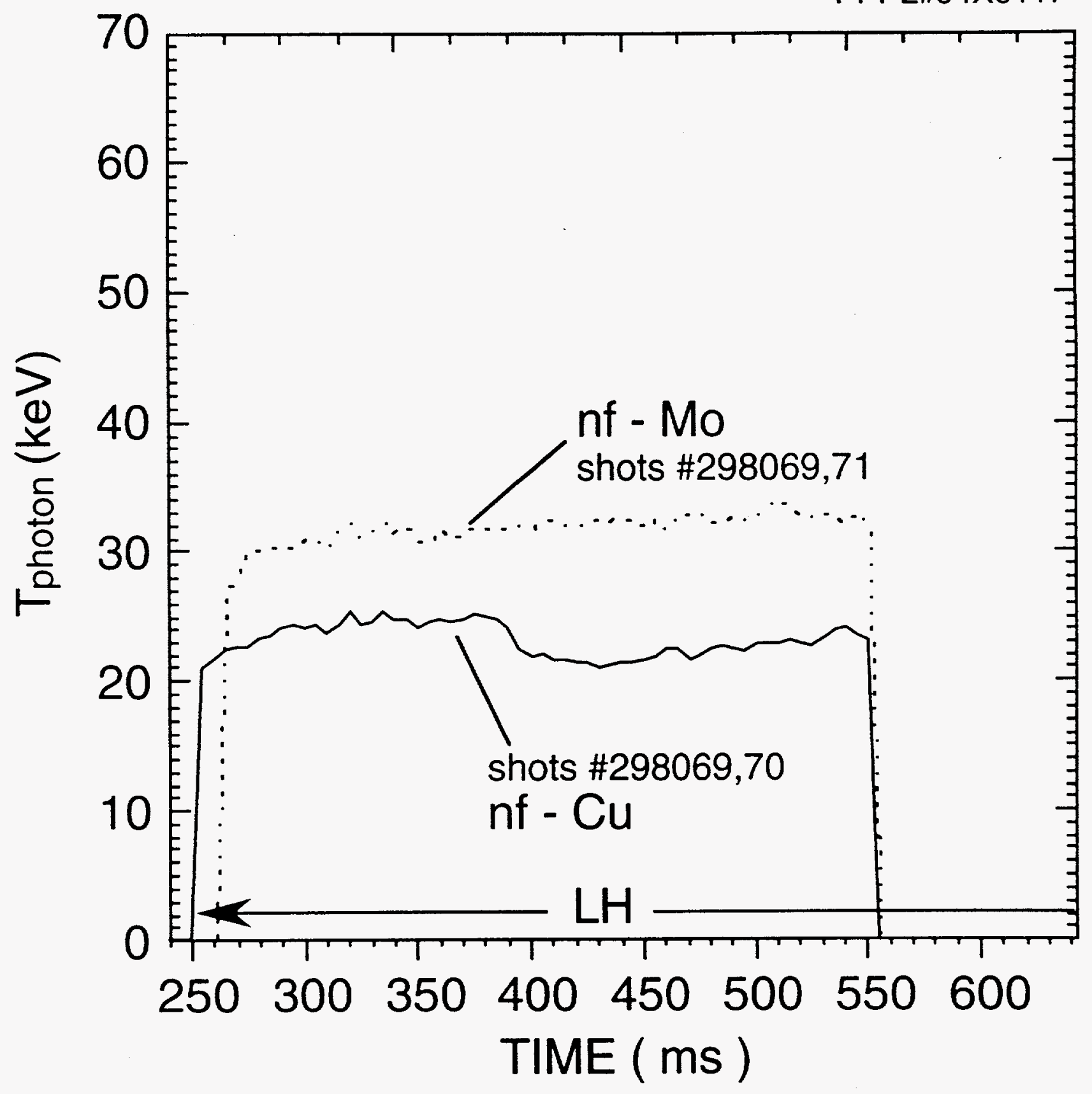

Fig. 25 


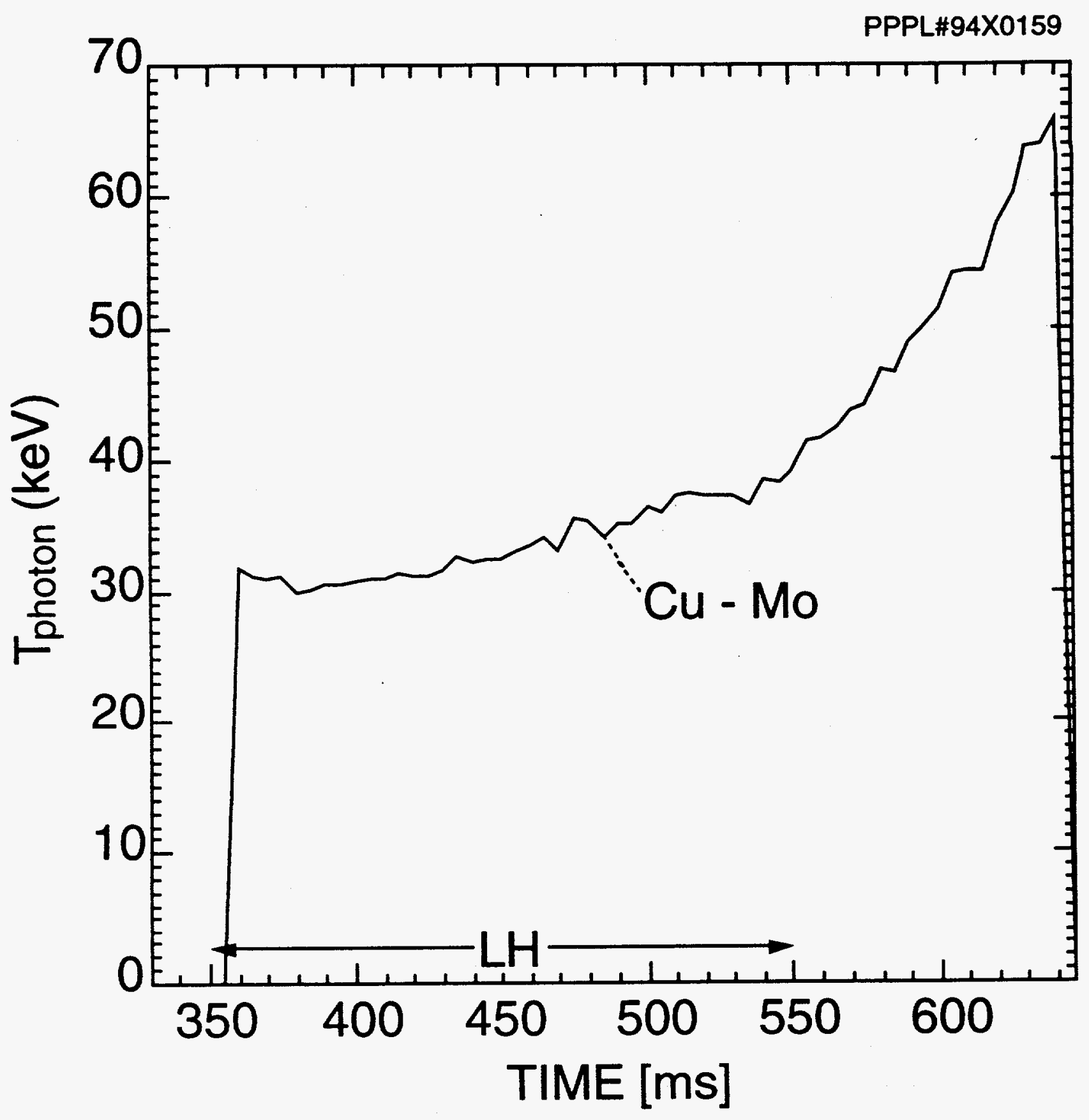

Fig. 26 
Dr. F. Paoloni, Univ. of Wollongong, AUSTRALIA

Prof. R.C. Cross, Univ. of Sydney, AUSTRALIA

Plasma Research Lab., Australian Nat. Univ., AUSTRALIA

Prof. I.R. Jones, Flinders Univ, AUSTRALIA

Prof. F. Cap, Inst. for Theoretical Physics, AUSTRIA

Prof. M. Heindler, Institut für Theoretische Physik, AUSTRIA

Prof. M. Goossens, Astronomisch Instituut, BELGIUM

Ecole Royale Militaire, Lab. de Phy. Plasmas, BELGIUM

Commission-European, DG. XII-Fusion Prog., BELGIUM

Prof. R. Bouciqué, Rijksuniversiteit Gent, BELGIUM

Dr. P.H. Sakanaka, Instituto Fisica, BRAZIL

Prof. Dr. I.C. Nascimento, Instituto Fisica, Sao Paulo, BRAZIL Instituto Nacional De Pesquisas Espaciais-INPE, BRAZIL Documents Office, Atomic Energy of Canada Ltd., CANADA Ms. M. Morin, CCFMTokamak de Varennes, CANADA Dr. M.P. Bachynski, MPB Technologies, Inc., CANADA Dr. H.M. Skarsgard, Univ. of Saskatchewan, CANADA Prof. J. Teichmann, Univ. of Montreal, CANADA

Prot. S.R. Sreenivasan, Univ, of Calgary, CANADA

Prof. T.W. Johnston, INAS-Energie, CANADA

Dr. R. Bolton, Centre canadien de fusion magnétique, CANADA

Dr. C.R. James, Univ. of Alberta, CANADA

Dr. P. Lukác, Komenského Universzita, CZECHO-SLOVAKIA

The Librarian, Culham Laboratory, ENGLAND

Library, R61, Rutherford Appleton Laboratory, ENGLAND

Mrs. S.A. Hutchinson, JET Library, ENGLAND

Dr. S.C. Sharma, Univ. of South Pacific, FIJI ISLANDS

P. Mähönen, Univ. of Helsinki, FINLAND

Prof. M.N. Bussac, Ecole Polytechnique,, FRANCE

C. Mouttet, Lab. de Physique des Milieux lonisés, FRANCE

J. Radet, CEN/CADARACHE - Bat 506, FRANCE

Prof. E. Economou, Univ. of Crete, GREECE

Ms. C. Rinni, Univ. of loannina, GREECE

Preprint Library, Hungarian Academy of Sci., HUNGARY

Dr. B. DasGupta, Saha Inst. of Nuclear Physics, INDIA

Dr. P. Kaw, Inst. for Plasma Research, INDIA

Dr. P. Rosenau, Israel Inst. of Technology, ISRAEL

Librarian, Intemational Center for Theo Physics, ITALY

Miss C. De Palo, Associazione EURATOM-ENEA, ITALY

Dr. G. Grosso, Istituto di Fisica del Plasma, ITALY

Prof. G. Rostangni, Istituto Gas lonizzati Del Cnr, ITALY
Dr. H. Yamato, Toshiba Res \& Devel Center, JAPAN

Prof. I. Kawakami, Hiroshima Univ., JAPAN

Prof. K. Nishikawa, Hiroshima Univ., JAPAN

Librarian, Naka Fusion Research Establishmem, JAERI, JAPAN

Director, Japan Atomic Energy Research Inst., JAPAN

Prof. S. Itoh, Kyushu Univ., JAPAN

Research Info. Ctr., National Instit. for Fusion Science, JAPAN

Prof. S. Tanaka, Kyoto Univ., JAPAN

Library, Kyoto Univ., JAPAN

Prof. N. Inoue, Univ. of Tokyo, JAPAN

Secretary, Plasma Section, Electrotechnical Lab., JAPAN

Dr. O. Mitarai, Kumamoto Inst. of Technology, JAPAN

Dr. G.S. Lee, Korea Basic Sci. Ctr., KOREA

J. Hyeon-Sook, Korea Atomic Energy Research Inst., KOREA

D.I. Choi, The Korea Adv. Inst. of Sci. \& Tech., KOREA

Prof. B.S. Liley, Univ. of Waikato, NEW ZEALAND

Inst of Physics, Chinese Acad Sci PEOPLE'S REP. OF CHINA

Library, Inst. of Plasma Physics, PEOPLE'S REP. OF CHINA

Tsinghua Univ. Library, PEOPLE'S REPUBLIC OF CHINA

Z. Li, S.W. Inst Physics, PEOPLE'S REPUBLIC OF CHINA

Prof. J.A.C. Cabral, Instituto Superior Tecnico, PORTUGAL

Prof. M.A. Hellberg, Univ. of Natal, S. AFRICA

Prof. D.E. Kim, Pohang Inst. of Sci. \& Tech., SO. KOREA

Prof. C.I.E.M.A.T, Fusion Division Library, SPAIN

Dr. L. Stenflo, Univ. of UMEA, SWEDEN

Library, Royal Inst. of Technology, SWEDEN

Prof. H. Wilhelmson, Chalmers Univ. of Tech., SWEDEN

Centre Phys. Des Plasmas, Ecole Polytech, SWITZERLAND

Bibliotheek, Inst. Voor Plasma-Fysica, THE NETHERLANDS

Asst. Prof. Dr. S. Cakir, Middle East Tech. Univ., TURKEY

Dr. V.A. Glukhikh,Sci. Res. Inst. Electrophys.I Apparatus, USSR

Dr. D.D. Ryutov, Siberian Branch of Academy of Sci., USSR

Dr. G.A. Eliseev, I.V. Kurchatov Inst., USSR

Librarian, The Ukr.SSR Academy of Sciences, USSR

Dr. L.M. Kovrizhnykh, Inst. of General Physics, USSR

Kernforschungsanlage GmbH, Zentralbibliothek, W. GERMANY Bibliothek, Inst. Für Plasmaforschung, W. GERMANY Prof. K. Schindler, Ruhr-Universitát Bochum, W. GERMANY

Dr. F. Wagner, (ASDEX), Max-Planck-Institut, W. GERMANY Librarian, Max-Planck-Institut, W. GERMANY 